\title{
Periodic Solutions of Some Polynomial Differential Systems in $\mathbb{R}^{4}$
}

\author{
Makhlouf Amar, Bousbiat Lilia \\ Department of Mathematics, University of Annaba, Annaba, Algeria \\ Email: amarmakhlouf@yahoo.fr, liliabst@gmail.com
}

How to cite this paper: Amar, M. and Lilia, B. (2017) Periodic Solutions of Some Polynomial Differential Systems in $\mathbb{R}^{4}$. Journal of Applied Mathematics and Physics, 5, 194-223.

http://dx.doi.org/10.4236/jamp.2017.51019

Received: December 20, 2016

Accepted: January 23, 2017

Published: January 26, 2017

Copyright $\odot 2017$ by authors and Scientific Research Publishing Inc. This work is licensed under the Creative Commons Attribution International License (CC BY 4.0).

http://creativecommons.org/licenses/by/4.0/

\section{Abstract}

We provide sufficient conditions for the existence of periodic solutions of the polynomial fourth order differential system

$\left(\begin{array}{c}\dot{x} \\ \dot{y} \\ \dot{u} \\ \dot{v}\end{array}\right)=\boldsymbol{A}\left(\begin{array}{l}x \\ y \\ u \\ v\end{array}\right)+\left(\begin{array}{l}h_{1}(t) \\ h_{2}(t) \\ h_{3}(t) \\ h_{4}(t)\end{array}\right)+\varepsilon\left(\begin{array}{l}P_{1}(x, y, u, v) \\ P_{2}(x, y, u, v) \\ P_{3}(x, y, u, v) \\ P_{4}(x, y, u, v)\end{array}\right)$, where $A$ is a $4 \times 4$ constant matrix, $P_{1}, P_{2}, P_{3}$ and $P_{4}$ are polynomials in the variables $x, y, u, v$ of degrees $n$, $h_{i}(t)=h_{i}(t+2 \pi)$ with $i=1,2,3,4$ being periodic functions and $\varepsilon$ is a small parameter.

\section{Keywords}

Periodic Solution, Averaging Theory, Differential System

\section{Introduction}

One of the main problems in the theory of differential systems is the study of their periodic orbits, their existence, their number and their stability. As usual a limit cycle of a differential system is a periodic orbit isolated in the set of all periodic orbits of the differential system.

The goal of this paper is to study the existence of the periodic orbits of the polynomial fourth order differential system

$$
\left(\begin{array}{c}
\dot{x} \\
\dot{y} \\
\dot{u} \\
\dot{v}
\end{array}\right)=\boldsymbol{A}\left(\begin{array}{c}
x \\
y \\
u \\
v
\end{array}\right)+\left(\begin{array}{l}
h_{1}(t) \\
h_{2}(t) \\
h_{3}(t) \\
h_{4}(t)
\end{array}\right)+\varepsilon\left(\begin{array}{l}
P_{1}(x, y, u, v) \\
P_{2}(x, y, u, v) \\
P_{3}(x, y, u, v) \\
P_{4}(x, y, u, v)
\end{array}\right),
$$

where $A$ is $4 \times 4$ a constant matrix, $P_{1}, P_{2}, P_{3}$ and $P_{4}$ are polynomials in the 
variables $x, y, u, v$ of degrees $n, h_{i}(t)=h_{i}(t+2 \pi)$ with $i=1,2,3,4$ being periodic functions and $\varepsilon$ is a small parameter.

There are many papers studying the periodic orbits of the fourth order differential systems and equations (see for instance [1]-[11]). But our main tool for studying the periodic orbits of the system (1.1) is completely different to the tools of the mentioned papers, and consequently the results obtained are distinct and new. We shall use the averaging theory, more precisely Theorem 6 and 7. Many of the quoted papers dealing with the peiodic orbits of four-order differential equations use Schauder's or Leray-Schauder's fixed point theorem, or the nonlocal reduction method or variational methods.

To obtain analytically periodic solutions is in general a very difficult work, usually impossible. Here with the averaging theory we reduce this difficult problem for the differential system (1.1) to find the zeros of a nonlinear system of four equations with four unknowns. It is known that in general the averaging theory for finding periodic solutions does not provide all the periodic solutions of the system. To explain this idea, there are two main reasons. First, the averaging theory for studying the periodic solutions of a differential system is based on the so-called displacement function, whose zeros provide periodic solutions. This displacement function in general is not global and consequently it cannot control all the periodic solution, only the ones which are in its domain of definition and that are hyperbolic. Second, the displacement function is expanded in power series of a small parameter $\varepsilon$, and the averaging theory only controls the zeros of the dominant term of this displacement function. When the dominant term is $\varepsilon^{k}$, we talk about the averaging theory of order $k$. For more details, see for instance [12] and the references quoted there.

The method of averaging is a classical tool that allows studying the dynamics of the nonlinear differential systems under periodic forcing. The method of averaging has a long history that starts with the classical works of Lagrange and Laplace, who provided an intuitive justification of the method. The first formalization of this theory was done in 1928 by Fatou [13]. Important practical and theoretical contributions to the averaging theory were made in the 1930's by Bogoliubov and Krylov [14], in 1945 by Bogoliubov [15], and by Bogoliubov and Mitropolsky [16] (English version 1961). For a more modern exposition of the averaging theory see the book of Sanders, Verhulst and Murdock [17]. For more information about averaging theory, see Section 2 of this paper.

In [18], the authors studied the bifurcation of limit cycles from the periodic orbits of a linear differential system in $\mathbb{R}^{4}$ in resonance $1: n$ perturbed inside a class of piecewise linear differential systems which appear in a natural way in control theory. In [19], the authors studied the limit cycles of the fourth-order differential equation

$$
\dddot{x}-(\lambda+\mu) \dddot{x}+(1+\lambda \mu) \ddot{x}-(\lambda+\mu) \dot{x}+\lambda \mu x=\varepsilon F(x, \dot{x}, \ddot{x}, \dddot{x}, t),
$$

where $\varepsilon$ is a small enough parameter and $F \in C^{2}$ is a $2 \pi$-periodic function in the variable $t$. In [20], the authors studied the autonomous case of the previous equation, (i.e. $F$ does not depend on $t$ ) using another approach. In [21], the 
authors provide sufficient conditions for the existence of periodic solutions of the fourth-order differential equation

$$
\dddot{x}+\left(1+p^{2}\right) \ddot{x}+p^{2} x=\varepsilon F(x, \dot{x}, \ddot{x}, \dddot{x}),
$$

where $p$ is a rational number different from $0, \varepsilon$ is small and $F$ is a nonlinear function. In [22], the authors provide sufficient conditions for the existence of periodic solutions of the fourth-order differential equation

$$
\dddot{x}+q \ddot{x}+p x=\varepsilon F(t, x, \dot{x}, \ddot{x}, \dddot{x}),
$$

where $p, q$ and $\varepsilon$ are real parameters, $\varepsilon$ is small and $F$ is a nonlinear nonautonomous periodic function with respect to $t$. The five previous cited papers used averaging method.

In [23] we studied the system (1.1) in dimension 3 using averaging method, i.e. the following system

$$
\begin{gathered}
\dot{x}=-y+\varepsilon P(x, y, z)+h_{1}(t), \\
\dot{y}=x+\varepsilon Q(x, y, z)+h_{2}(t), \\
\dot{z}=a z+\varepsilon R(x, y, z)+h_{3}(t),
\end{gathered}
$$

where $a$ is a real number, $P, Q$ and $R$ are polynomials in the variables $x, y, z$ of degrees $n, h_{i}(t)=h_{i}(t+2 \pi)$ with $i=1,2,3$ being periodic functions and $\varepsilon$ is a small parameter. In this paper our objective is to provide the existence of periodic solutions of system (1.1).

Our main results on the periodic solutions of the differential system (1.1) are the following theorems.

One considers system (1.1) with $\boldsymbol{A}=\left(\begin{array}{cccc}0 & -1 & 0 & 0 \\ 1 & 0 & 0 & 0 \\ 0 & 0 & 0 & -1 \\ 0 & 0 & 1 & 0\end{array}\right)$, our result is the following.

Theorem 1. One defines

$$
\begin{aligned}
& \mathcal{F}_{1}\left(x_{0}, y_{0}, u_{0}, v_{0}\right) \\
& =\frac{1}{2 \pi} \int_{0}^{2 \pi}\left(\cos (t) P_{1}(a(t), b(t), c(t), d(t))+\sin (t) P_{2}(a(t), b(t), c(t), d(t))\right) \mathrm{d} t, \\
& \mathcal{F}_{2}\left(x_{0}, y_{0}, u_{0}, v_{0}\right) \\
& =\frac{1}{2 \pi} \int_{0}^{2 \pi}\left(-\sin (t) P_{1}(a(t), b(t), c(t), d(t))+\cos (t) P_{2}(a(t), b(t), c(t), d(t))\right) \mathrm{d} t, \\
& \mathcal{F}_{3}\left(x_{0}, y_{0}, u_{0}, v_{0}\right) \\
& =\frac{1}{2 \pi} \int_{0}^{2 \pi}\left(\cos (t) P_{3}(a(t), b(t), c(t), d(t))+\sin (t) P_{4}(a(t), b(t), c(t), d(t))\right) \mathrm{d} t, \\
& \mathcal{F}_{4}\left(x_{0}, y_{0}, u_{0}, v_{0}\right) \\
& =\frac{1}{2 \pi} \int_{0}^{2 \pi}\left(-\sin (t) P_{3}(a(t), b(t), c(t), d(t))+\cos (t) P_{4}(a(t), b(t), c(t), d(t))\right) \mathrm{d} t,
\end{aligned}
$$

where

$$
a(t)=\cos (t) x_{0}-\sin (t) y_{0}+\int_{0}^{t}\left(\cos (t-s) h_{1}(s)-\sin (t-s) h_{2}(s)\right) \mathrm{d} s,
$$




$$
\begin{aligned}
& b(t)=\sin (t) x_{0}+\cos (t) y_{0}+\int_{0}^{t}\left(\sin (t-s) h_{1}(s)+\cos (t-s) h_{2}(s)\right) \mathrm{d} s, \\
& c(t)=\cos (t) u_{0}-\sin (t) v_{0}+\int_{0}^{t}\left(\cos (t-s) h_{3}(s)-\sin (t-s) h_{4}(s)\right) \mathrm{d} s, \\
& d(t)=\sin (t) u_{0}+\cos (t) v_{0}+\int_{0}^{t}\left(\sin (t-s) h_{3}(s)+\cos (t-s) h_{4}(s)\right) \mathrm{d} s .
\end{aligned}
$$

If

$$
\begin{aligned}
& \int_{0}^{2 \pi}\left(\cos (s) h_{1}(s)-\sin (s) h_{2}(s)\right) \mathrm{d} s=0, \\
& \int_{0}^{2 \pi}\left(-\sin (s) h_{1}(s)+\cos (s) h_{2}(s)\right) \mathrm{d} s=0, \\
& \int_{0}^{2 \pi}\left(\cos (s) h_{3}(s)-\sin (s) h_{4}(s)\right) \mathrm{d} s=0, \\
& \int_{0}^{2 \pi}\left(-\sin (s) h_{3}(s)+\cos (s) h_{4}(s)\right) \mathrm{d} s=0,
\end{aligned}
$$

then for every $\left(x_{0}^{*}, y_{0}^{*}, u_{0}^{*}, v_{0}^{*}\right)$ solution of the system

$$
\mathcal{F}_{k}\left(x_{0}, y_{0}, u_{0}, v_{0}\right)=0, \quad k=1,2,3,4,
$$

satisfying

$$
\operatorname{det}\left(\left.\frac{\partial\left(\mathcal{F}_{1}, \mathcal{F}_{2}, \mathcal{F}_{3}, \mathcal{F}_{4}\right)}{\partial\left(x_{0}, y_{0}, u_{0}, v_{0}\right)}\right|_{\left(x_{0}, y_{0}, u_{0}, v_{0}\right)=\left(x_{0}^{*}, y_{0}^{*}, u_{0}^{*}, v_{0}^{*}\right)}\right) \neq 0,
$$

the differential system (1.1) has a periodic solution $\left(\begin{array}{l}x(t, \varepsilon) \\ y(t, \varepsilon) \\ u(t, \varepsilon) \\ v(t, \varepsilon)\end{array}\right)$, which tends to the periodic solution given by

$$
\left(\begin{array}{l}
x(t) \\
y(t) \\
u(t) \\
v(t)
\end{array}\right)=\left(\begin{array}{l}
\cos (t) x_{0}^{*}-\sin (t) y_{0}^{*}+\int_{0}^{t}\left(\cos (t-s) h_{1}(s)-\sin (t-s) h_{2}(s)\right) \mathrm{d} s \\
\sin (t) x_{0}^{*}+\cos (t) y_{0}^{*}+\int_{0}^{t}\left(\sin (t-s) h_{1}(s)+\cos (t-s) h_{2}(s)\right) \mathrm{d} s \\
\cos (t) u_{0}^{*}-\sin (t) v_{0}^{*}+\int_{0}^{t}\left(\cos (t-s) h_{3}(s)-\sin (t-s) h_{4}(s)\right) \mathrm{d} s \\
\sin (t) u_{0}^{*}+\cos (t) v_{0}^{*}+\int_{0}^{t}\left(\sin (t-s) h_{3}(s)+\cos (t-s) h_{4}(s)\right) \mathrm{d} s
\end{array}\right)
$$

of the differential system

$$
\begin{gathered}
\dot{x}=-y+h_{1}(t), \\
\dot{y}=x+h_{2}(t), \\
\dot{u}=-v+h_{3}(t), \\
\dot{v}=u+h_{4}(t),
\end{gathered}
$$

when $\varepsilon \rightarrow 0$.

Note that this solution is periodic of period $2 \pi$.

One considers system (1.1) with $\boldsymbol{A}=\left(\begin{array}{cccc}0 & -1 & 0 & 0 \\ 1 & 0 & 0 & 0 \\ 0 & 0 & \lambda & 0 \\ 0 & 0 & 0 & \mu\end{array}\right)$. We distinguish three 
cases for different parameter values $\lambda$ and $\mu$ :

Case 1: $\lambda \neq 0$ and $\mu \neq 0$.

Case 2: $\lambda=0$ and $\mu \neq 0$. (Or $\lambda \neq 0$ and $\mu=0$ ).

Case 3: $\lambda=\mu=0$.

Our results for these three cases are the following ones.

Theorem 2. Case 1

One defines

$$
\begin{aligned}
& \mathcal{F}_{1}\left(x_{0}, y_{0}\right) \\
& =\frac{1}{2 \pi} \int_{0}^{2 \pi}\left(\cos (t) P_{1}(a(t), b(t), c(t), d(t))+\sin (t) P_{2}(a(t), b(t), c(t), d(t))\right) \mathrm{d} t, \\
& \mathcal{F}_{2}\left(x_{0}, y_{0}\right) \\
& =\frac{1}{2 \pi} \int_{0}^{2 \pi}\left(-\sin (t) P_{1}(a(t), b(t), c(t), d(t))+\cos (t) P_{2}(a(t), b(t), c(t), d(t))\right) \mathrm{d} t, \\
& \text { where } \\
& \qquad a(t)=\cos (t) x_{0}-\sin (t) y_{0}+\int_{0}^{t}\left(\cos (t-s) h_{1}(s)-\sin (t-s) h_{2}(s)\right) \mathrm{d} s, \\
& \qquad b(t)=\sin (t) x_{0}+\cos (t) y_{0}+\int_{0}^{t}\left(\sin (t-s) h_{1}(s)+\cos (t-s) h_{2}(s)\right) \mathrm{d} s, \\
& \qquad c(t)=e^{\lambda t} u_{0}+\int_{0}^{t} e^{\lambda(t-s)} h_{3}(s) \mathrm{d} s, \\
& \qquad d(t)=e^{\mu t} v_{0}+\int_{0}^{t} e^{\mu(t-s)} h_{4}(s) \mathrm{d} s .
\end{aligned}
$$

If

$$
\begin{gathered}
\int_{0}^{2 \pi}\left(\cos (s) h_{1}(s)+\sin (s) h_{2}(s)\right) \mathrm{d} s=0, \\
\int_{0}^{2 \pi}\left(-\sin (s) h_{1}(s)+\cos (s) h_{2}(s)\right) \mathrm{d} s=0, \\
u_{0}=\frac{1}{1-e^{2 \pi \lambda}} \int_{0}^{2 \pi} e^{\lambda(2 \pi-s)} h_{3}(s) \mathrm{d} s, \\
v_{0}=\frac{1}{1-e^{2 \pi \mu}} \int_{0}^{2 \pi} e^{\mu(2 \pi-s)} h_{4}(s) \mathrm{d} s,
\end{gathered}
$$

then for every $\left(x_{0}^{*}, y_{0}^{*}\right)$ solution of the system

$$
\mathcal{F}_{k}\left(x_{0}, y_{0}\right)=0, k=1,2,
$$

satisfying

$$
\operatorname{det}\left(\left.\frac{\partial\left(\mathcal{F}_{1}, \mathcal{F}_{2}\right)}{\partial\left(x_{0}, y_{0}\right)}\right|_{\left(x_{0}, y_{0}\right)=\left(x_{0}^{*}, y_{0}^{*}\right)}\right) \neq 0,
$$

the differential system (1.1) has a periodic solution $\left(\begin{array}{l}x(t, \varepsilon) \\ y(t, \varepsilon) \\ u(t, \varepsilon) \\ v(t, \varepsilon)\end{array}\right)$, which tends to the periodic solution given by 


$$
\left(\begin{array}{l}
x(t) \\
y(t) \\
u(t) \\
v(t)
\end{array}\right)=\left(\begin{array}{c}
\cos (t) x_{0}^{*}-\sin (t) y_{0}^{*}+\int_{0}^{t}\left(\cos (t-s) h_{1}(s)-\sin (t-s) h_{2}(s)\right) \mathrm{d} s \\
\sin (t) x_{0}^{*}+\cos (t) y_{0}^{*}+\int_{0}^{t}\left(\sin (t-s) h_{1}(s)+\cos (t-s) h_{2}(s)\right) \mathrm{d} s \\
\frac{e^{\lambda t}}{1-e^{2 \pi \lambda}} \int_{0}^{2 \pi} e^{\lambda(2 \pi-s)} h_{3}(s) \mathrm{d} s+\int_{0}^{t} e^{\lambda(t-s)} h_{3}(s) \mathrm{d} s \\
\frac{e^{\mu t}}{1-e^{2 \pi \mu}} \int_{0}^{2 \pi} e^{\mu(2 \pi-s)} h_{3}(s) \mathrm{d} s+\int_{0}^{t} e^{\mu(t-s)} h_{4}(s) \mathrm{d} s
\end{array}\right)
$$

of the differential system

$$
\begin{gathered}
\dot{x}=-y+h_{1}(t), \\
\dot{y}=x+h_{2}(t), \\
\dot{u}=\lambda u+h_{3}(t), \\
\dot{v}=\mu v+h_{4}(t),
\end{gathered}
$$

when $\varepsilon \rightarrow 0$.

Note that this solution is periodic of period $2 \pi$.

Theorem 3. Case $2(\lambda=0)$

One defines

$\mathcal{F}_{1}\left(x_{0}, y_{0}, u_{0}\right)$

$=\frac{1}{2 \pi} \int_{0}^{2 \pi}\left(\cos (t) P_{1}(a(t), b(t), c(t), d(t))+\sin (t) P_{2}(a(t), b(t), c(t), d(t))\right) \mathrm{d} t$,

$\mathcal{F}_{2}\left(x_{0}, y_{0}, u_{0}\right)$

$=\frac{1}{2 \pi} \int_{0}^{2 \pi}\left(-\sin (t) P_{1}(a(t), b(t), c(t), d(t))+\cos (t) P_{2}(a(t), b(t), c(t), d(t))\right) \mathrm{d} t$,

$$
\mathcal{F}_{3}\left(x_{0}, y_{0}, u_{0}\right)=\frac{1}{2 \pi} \int_{0}^{2 \pi} P_{3}(a(t), b(t), c(t), d(t)) \mathrm{d} t,
$$

where

$$
\begin{gathered}
a(t)=\cos (t) x_{0}-\sin (t) y_{0}+\int_{0}^{t}\left(\cos (t-s) h_{1}(s)-\sin (t-s) h_{2}(s)\right) \mathrm{d} s \\
b(t)=\sin (t) x_{0}+\cos (t) y_{0}+\int_{0}^{t}\left(\sin (t-s) h_{1}(s)+\cos (t-s) h_{2}(s)\right) \mathrm{d} s, \\
c(t)=u_{0}+\int_{0}^{t} h_{3}(s) \mathrm{d} s, \\
d(t)=e^{\mu t} v_{0}+\int_{0}^{t} e^{\mu(t-s)} h_{4}(s) \mathrm{d} s .
\end{gathered}
$$

If

$$
\begin{aligned}
& \int_{0}^{2 \pi}\left(\cos (s) h_{1}(s)+\sin (s) h_{2}(s)\right) \mathrm{d} s=0, \\
& \int_{0}^{2 \pi}\left(-\sin (s) h_{1}(s)+\cos (s) h_{2}(s)\right) \mathrm{d} s=0, \\
& \int_{0}^{2 \pi} h_{3}(s) \mathrm{d} s=0, \\
& v_{0}=\frac{1}{1-e^{2 \pi \mu}} \int_{0}^{2 \pi} e^{\mu(2 \pi-s)} h_{4}(s) \mathrm{d} s,
\end{aligned}
$$

then for every $\left(x_{0}^{*}, y_{0}^{*}, u_{0}^{*}\right)$ solution of the system

$$
\mathcal{F}_{k}\left(x_{0}, y_{0}, u_{0}\right)=0, k=1,2,3,
$$


satisfying

$$
\operatorname{det}\left(\left.\frac{\partial\left(\mathcal{F}_{1}, \mathcal{F}_{2}, \mathcal{F}_{3}\right)}{\partial\left(x_{0}, y_{0}, u_{0}\right)}\right|_{\left(x_{0}, y_{0}, u_{0}\right)=\left(x_{0}^{*}, y_{0}^{*}, u_{0}^{*}\right)}\right) \neq 0,
$$

the differential system (1.1) has a periodic solution $\left(\begin{array}{l}x(t, \varepsilon) \\ y(t, \varepsilon) \\ u(t, \varepsilon) \\ v(t, \varepsilon)\end{array}\right)$, which tends to the periodic solution given by

$$
\left(\begin{array}{c}
x(t) \\
y(t) \\
u(t) \\
v(t)
\end{array}\right)=\left(\begin{array}{c}
\cos (t) x_{0}^{*}-\sin (t) y_{0}^{*}+\int_{0}^{t}\left(\cos (t-s) h_{1}(s)-\sin (t-s) h_{2}(s)\right) \mathrm{d} s \\
\sin (t) x_{0}^{*}+\cos (t) y_{0}^{*}+\int_{0}^{t}\left(\sin (t-s) h_{1}(s)+\cos (t-s) h_{2}(s)\right) \mathrm{d} s \\
u_{0}^{*}+\int_{0}^{t} h_{3}(s) \mathrm{d} s \\
\frac{e^{\mu t}}{1-e^{2 \pi \mu}} \int_{0}^{2 \pi} e^{\mu(2 \pi-s)} h_{4}(s) \mathrm{d} s+\int_{0}^{t} e^{\mu(t-s)} h_{4}(s) \mathrm{d} s
\end{array}\right)
$$

of the differential system

$$
\begin{gathered}
\dot{x}=-y+h_{1}(t), \\
\dot{y}=x+h_{2}(t), \\
\dot{u}=h_{3}(t), \\
\dot{v}=\mu v+h_{4}(t),
\end{gathered}
$$

when $\varepsilon \rightarrow 0$.

Note that this solution is periodic of period $2 \pi$.

Theorem 4. Case 3

One defines

$$
\begin{gathered}
\mathcal{F}_{1}\left(x_{0}, y_{0}, u_{0}, v_{0}\right) \\
=\frac{1}{2 \pi} \int_{0}^{2 \pi}\left(\cos (t) P_{1}(a(t), b(t) c(t), d(t))+\sin (t) P_{2}(a(t), b(t), c(t), d(t))\right) \mathrm{d} t, \\
\mathcal{F}_{2}\left(x_{0}, y_{0}, u_{0}, v_{0}\right) \\
=\frac{1}{2 \pi} \int_{0}^{2 \pi}\left(-\sin (t) P_{1}(a(t), b(t), c(t), d(t))+\cos (t) P_{2}(a(t), b(t), c(t), d(t))\right) \mathrm{d} t, \\
\mathcal{F}_{3}\left(x_{0}, y_{0}, u_{0}, v_{0}\right)=\frac{1}{2 \pi} \int_{0}^{2 \pi}\left(P_{3}(a(t), b(t), c(t), d(t))\right) \mathrm{d} t, \\
\mathcal{F}_{3}\left(x_{0}, y_{0}, u_{0}, v_{0}\right)=\frac{1}{2 \pi} \int_{0}^{2 \pi}\left(P_{4}(a(t), b(t), c(t), d(t))\right) \mathrm{d} t,
\end{gathered}
$$

where

$$
\begin{gathered}
a(t)=\cos (t) x_{0}-\sin (t) y_{0}+\int_{0}^{t}\left(\cos (t-s) h_{1}(s)-\sin (t-s) h_{2}(s)\right) \mathrm{d} s, \\
b(t)=\sin (t) x_{0}+\cos (t) y_{0}+\int_{0}^{t}\left(\sin (t-s) h_{1}(s)+\cos (t-s) h_{2}(s)\right) \mathrm{d} s, \\
c(t)=u_{0}+\int_{0}^{t} h_{3}(s) \mathrm{d} s,
\end{gathered}
$$




$$
d(t)=v_{0}+\int_{0}^{t} h_{4}(s) d s
$$

If

$$
\begin{aligned}
& \int_{0}^{2 \pi}\left(\cos (s) h_{1}(s)+\sin (s) h_{2}(s)\right) \mathrm{d} s=0, \\
& \int_{0}^{2 \pi}\left(-\sin (s) h_{1}(s)+\cos (s) h_{2}(s)\right) \mathrm{d} s=0, \\
& \int_{0}^{2 \pi} h_{3}(s) \mathrm{d} s=0, \\
& \int_{0}^{2 \pi} h_{4}(s) \mathrm{d} s=0,
\end{aligned}
$$

then for every $\left(x_{0}^{*}, y_{0}^{*}, u_{0}^{*}, v_{0}^{*}\right)$ solution of the system

$$
\mathcal{F}_{k}\left(x_{0}, y_{0}, u_{0}, v_{0}\right)=0, k=1,2,3,4,
$$

satisfying

$$
\operatorname{det}\left(\left.\frac{\partial\left(\mathcal{F}_{1}, \mathcal{F}_{2}, \mathcal{F}_{3}, \mathcal{F}_{4}\right)}{\partial\left(x_{0}, y_{0}, u_{0}, v_{0}\right)}\right|_{\left(x_{0}, y_{0}, u_{0}, v_{0}\right)=\left(x_{0}^{*}, y_{0}^{*}, u_{0}^{*}, v_{0}^{*}\right)}\right) \neq 0,
$$

the differential system (1.1) has a periodic solution $\left(\begin{array}{l}x(t, \varepsilon) \\ y(t, \varepsilon) \\ u(t, \varepsilon) \\ v(t, \varepsilon)\end{array}\right)$, which tends to the periodic solution given by

$$
\left(\begin{array}{l}
x(t) \\
y(t) \\
u(t) \\
v(t)
\end{array}\right)=\left(\begin{array}{l}
\cos (t) x_{0}^{*}-\sin (t) y_{0}^{*}+\int_{0}^{t}\left(\cos (t-s) h_{1}(s)-\sin (t-s) h_{2}(s)\right) \mathrm{d} s \\
\sin (t) x_{0}^{*}+\cos (t) y_{0}^{*}+\int_{0}^{t}\left(\sin (t-s) h_{1}(s)+\cos (t-s) h_{2}(s)\right) \mathrm{d} s \\
u_{0}^{*}+\int_{0}^{t} h_{3}(s) \mathrm{d} s \\
v_{0}^{*}+\int_{0}^{t} h_{4}(s) \mathrm{d} s
\end{array}\right)
$$

of the differential system

$$
\begin{gathered}
\dot{x}=-y+h_{1}(t), \\
\dot{y}=x+h_{2}(t), \\
\dot{u}=h_{3}(t), \\
\dot{v}=h_{4}(t),
\end{gathered}
$$

when $\varepsilon \rightarrow 0$.

Note that this solution is periodic of period $2 \pi$.

One considers system (1.1) with $\boldsymbol{A}=\left(\begin{array}{cccc}0 & -1 & 0 & 0 \\ 1 & 0 & 0 & 0 \\ 0 & 0 & \lambda & 1 \\ 0 & 0 & 0 & \lambda\end{array}\right)$. Our result are the following.

Theorem 5. One defines

$$
\begin{aligned}
& \mathcal{F}_{1}\left(x_{0}, y_{0}\right) \\
& =\frac{1}{2 \pi} \int_{0}^{2 \pi}\left(\cos (t) P_{1}(a(t), b(t), c(t), d(t))+\sin (t) P_{2}(a(t), b(t), c(t), d(t))\right) \mathrm{d} t,
\end{aligned}
$$




$$
\begin{aligned}
& \mathcal{F}_{2}\left(x_{0}, y_{0}\right) \\
& =\frac{1}{2 \pi} \int_{0}^{2 \pi}\left(-\sin (t) P_{1}(a(t), b(t), c(t), d(t))+\cos (t) P_{2}(a(t), b(t), c(t), d(t))\right) \mathrm{d} t, \\
& \text { where } \\
& \qquad a(t)=\cos (t) x_{0}-\sin (t) y_{0}+\int_{0}^{t}\left(\cos (t-s) h_{1}(s)-\sin (t-s) h_{2}(s)\right) \mathrm{d} s, \\
& \qquad b(t)=\sin (t) x_{0}+\cos (t) y_{0}+\int_{0}^{t}\left(\sin (t-s) h_{1}(s)+\cos (t-s) h_{2}(s)\right) \mathrm{d} s, \\
& \qquad c(t)=e^{\lambda t} u_{0}+t e^{\lambda t} v_{0}+\int_{0}^{t} e^{\lambda(t-s)}\left(h_{3}(s)+(t-s) h_{4}(s)\right) \mathrm{d} s, \\
& \qquad d(t)=e^{\lambda t} v_{0}+\int_{0}^{t} e^{\lambda(t-s)} h_{4}(s) \mathrm{d} s .
\end{aligned}
$$

If

$$
\begin{aligned}
& \int_{0}^{2 \pi}\left(\cos (s) h_{1}(s)+\sin (s) h_{2}(s)\right) \mathrm{d} s=0, \\
& \int_{0}^{2 \pi}\left(-\sin (s) h_{1}(s)+\cos (s) h_{2}(s)\right) \mathrm{d} s=0, \\
& u_{0}=\frac{2 \pi}{\left(1-e^{2 \pi \lambda}\right)^{2}} \int_{0}^{2 \pi} e^{\lambda(2 \pi-s)} h_{4}(s) \mathrm{d} s+\frac{1}{1-e^{2 \pi \lambda}} \int_{0}^{2 \pi} e^{\lambda(2 \pi-s)}\left(h_{3}(s)-s h_{4}(s)\right) \mathrm{d} s, \\
& v_{0}=\frac{1}{1-e^{2 \pi \lambda}} \int_{0}^{2 \pi} e^{\lambda(2 \pi-s)} h_{4}(s) \mathrm{d} s,
\end{aligned}
$$

then for every $\left(x_{0}^{*}, y_{0}^{*}\right)$ solution of the system

$$
\mathcal{F}_{k}\left(x_{0}, y_{0}\right)=0, k=1,2,
$$

satisfying

$$
\operatorname{det}\left(\left.\frac{\partial\left(\mathcal{F}_{1}, \mathcal{F}_{2}\right)}{\partial\left(x_{0}, y_{0}\right)}\right|_{\left(x_{0}, y_{0}\right)=\left(x_{0}^{*}, y_{0}^{*}\right)}\right) \neq 0,
$$

the differential system (1) has a periodic solution $\left(\begin{array}{c}x(t, \varepsilon) \\ y(t, \varepsilon) \\ u(t, \varepsilon) \\ v(t, \varepsilon)\end{array}\right)$, which tends to the periodic solution given by

$$
\left(\begin{array}{l}
x(t) \\
y(t) \\
u(t) \\
v(t)
\end{array}\right)=\left(\begin{array}{c}
\cos (t) x_{0}^{*}-\sin (t) y_{0}^{*}+\int_{0}^{t}\left(\cos (t-s) h_{1}(s)-\sin (t-s) h_{2}(s)\right) \mathrm{d} s \\
\sin (t) x_{0}^{*}+\cos (t) y_{0}^{*}+\int_{0}^{t}\left(\sin (t-s) h_{1}(s)+\cos (t-s) h_{2}(s)\right) \mathrm{d} s \\
\int_{0}^{t} e^{\lambda(t-s)}\left(h_{3}(s)+(t-s) h_{4}(s)\right) \mathrm{d} s+\frac{e^{2 \pi \lambda} \int_{0}^{2 \pi} e^{\lambda(t-s)}\left(h_{3}(s)-s h_{4}(s)\right) \mathrm{d} s}{e^{2 \pi \lambda}-1} \\
+\frac{2 \pi e^{2 \pi \lambda} \int_{0}^{2 \pi} e^{\lambda(t-s)} h_{4}(s) \mathrm{d} s}{\left(e^{2 \pi \lambda}-1\right)^{2}} \\
\frac{e^{2 \pi \lambda}}{1-e^{2 \pi \lambda}} \int_{0}^{2 \pi} e^{\lambda(t-s)} h_{4}(s) \mathrm{d} s+\int_{0}^{t} e^{\lambda(t-s)} h_{4}(s) \mathrm{d} s
\end{array}\right)
$$

of the differential system 


$$
\begin{gathered}
\dot{x}=-y+h_{1}(t), \\
\dot{y}=x+h_{2}(t), \\
\dot{u}=\lambda u+v+h_{3}(t), \\
\dot{v}=\lambda v+h_{4}(t),
\end{gathered}
$$

when $\varepsilon \rightarrow 0$.

Note that this solution is periodic of period $2 \pi$.

\section{Basic Results on Averaging Theory}

In this section we present the basic results on the averaging theory that we shall need for proving the main results of this paper.

We consider the problem of the bifurcation of T-periodic solutions from differential systems of the form

$$
\dot{x}=F_{0}(t, x)+\varepsilon F_{1}(t, x)+\varepsilon^{2} F_{2}(t, x, \varepsilon) .
$$

with $\varepsilon=0$ to $\varepsilon \neq 0$ being sufficiently small. Here the functions

$F_{0}, F_{1}: \mathbb{R} \times \Omega \rightarrow \mathbb{R}^{n}$ and $F_{2}: \mathbb{R} \times \Omega \times\left(-\varepsilon_{0}, \varepsilon_{0}\right) \rightarrow \mathbb{R}^{n}$ are $C^{2}$ functions, T-periodic in the first variable, and $\Omega$ is an open subset of $\mathbb{R}^{n}$. The main assumption is that the unperturbed system

$$
\dot{\boldsymbol{x}}=F_{0}(t, \boldsymbol{x}),
$$

has a submanifold of periodic solutions. A solution of this problem is given using the averaging theory. For a general introduction to the averaging theory see the books of Sanders and Verhulst [17], and of Verhulst [24].

Let $\boldsymbol{x}(t, \mathbf{z}, \varepsilon)$ be the solution of the system (2.2) such that $\boldsymbol{x}(0, \mathbf{z}, \varepsilon)=\mathbf{z}$. We write the linearization of the unperturbed system along a periodic solution $\boldsymbol{x}(t, \mathbf{z}, 0)$ as

$$
\dot{\boldsymbol{y}}=D_{\boldsymbol{x}} F_{0}(t, \boldsymbol{x}(t, \mathbf{z}, 0)) \boldsymbol{y} .
$$

In what follows we denote by $M_{z}(t)$ some fundamental matrix of the linear differential system (2.2), and by $\xi: \mathbb{R}^{k} \times \mathbb{R}^{n-k} \rightarrow \mathbb{R}^{k}$ the projection of $\mathbb{R}^{n}$ onto its first $k$ coordinates; i.e. $\xi\left(x_{1}, \cdots, x_{n}\right)=\left(x_{1}, \cdots, x_{k}\right)$. We assume that there exists a $k$-dimensional submanifold $\mathbf{Z}$ of $\Omega$ filled with T-periodic solutions of (2.2). Then an answer to the problem of bifurcation of T-periodic solutions from the periodic solutions contained in $\mathbf{Z}$ for system (2.1) is given in the following result.

Theorem 6. Let $W$ be an open and bounded subset of $\mathbb{R}^{k}$, and let $\beta: C l(W) \rightarrow \mathbb{R}^{n-k}$ be a $C^{2}$ function. We assume that

(i) $\mathbf{Z}=\left\{\mathbf{z}_{\alpha}=(\alpha, \beta(\alpha)), \alpha \in C l(W)\right\} \subset \Omega$ and that for each $\mathbf{z}_{\alpha} \in \mathbf{Z}$ the solution $\mathbf{x}\left(t, \mathbf{z}_{\alpha}\right)$ of $(8)$ is T-periodic;

(ii) for each $\mathbf{z}_{\alpha} \in \mathbf{Z}$ there is a fundamental matrix $\boldsymbol{M}_{\boldsymbol{z}_{\alpha}}(t)$ of (9) such that the matrix $\boldsymbol{M}_{z_{\alpha}}^{-1}(0)-\boldsymbol{M}_{z_{\alpha}}^{-1}(T)$ has in the upper right corner the $k \times(n-k)$ zero matrix, and in the lower right corner a $(n-k) \times(n-k)$ matrix $\Delta_{\alpha}$ with $\operatorname{det}\left(\Delta_{\alpha}\right) \neq 0$. 
We consider the function $\mathcal{F}: C l(W) \rightarrow \mathbb{R}^{k}$

$$
\mathcal{F}(\boldsymbol{\alpha})=\xi\left(\frac{1}{T} \int_{0}^{T} \boldsymbol{M}_{\mathbf{z}_{\alpha}}^{-1}(t) F_{1}\left(t, \boldsymbol{x}\left(t, \mathbf{z}_{\alpha}\right)\right) \mathrm{d} t\right) .
$$

If there exists $a \in W$ with $\mathcal{F}(a)=0$ and $\operatorname{det}((\mathrm{d} \mathcal{F} / \mathrm{d} \alpha)(a)) \neq 0$, then there is a T-periodic solution $\varphi(t, \varepsilon)$ of system (2.1) such that $\varphi(0, \varepsilon) \rightarrow \mathbf{z}_{a}$ as $\varepsilon \rightarrow 0$.

Theorem 6 goes back to Malkin [25] and Roseau [26], for a shorter proof see [27].

We assume that there exists an open set $V$ with $\mathrm{Cl}(V) \subset \Omega$ such that for each $\mathbf{z} \in C l(V), \quad \boldsymbol{x}(t, \boldsymbol{z}, 0)$ is T-periodic, where $\boldsymbol{x}(t, \mathbf{z}, 0)$ denotes the solution of the unperturbed system (2.2) with $\boldsymbol{x}(0, \boldsymbol{z}, 0)=\mathbf{z}$. The set $C l(V)$ is isochronous for the system (2.1); i.e. it is a set formed only by periodic orbits, all of them having the same period. Then, an answer to the problem of the bifurcation of T-periodic solutions from the periodic solutions $x(t, z, 0)$ contained in $\mathrm{Cl}(\mathrm{V})$ is given in the following result.

\section{Theorem 7. (Perturbations of an isochronous set)}

We assume that there exists an open and bounded set $V$ with $\mathrm{Cl}(V) \subset \Omega$ such that for each $\mathbf{z} \in C l(V)$, the solution $\mathbf{x}(t, \mathbf{z}, 0)$ is T-periodic, considering a function $\mathcal{F}: C l(V) \rightarrow \mathbb{R}^{n}$ defined by

$$
\mathcal{F}(\mathbf{z})=\int_{0}^{T} M_{\mathbf{z}}^{-1}(t) F_{1}(t, \boldsymbol{x}(t, \mathbf{z})) \mathrm{d} t .
$$

If there exists an $a \in V$ with $\mathcal{F}(a)=0$ and $\operatorname{det}\left(\left(\frac{\partial \mathcal{F}}{\partial \mathbf{z}}\right)(a)\right) \neq 0$, then there exists a T-periodic solution $\varphi(t, \varepsilon)$ to system (2.1) such that $\varphi(0, \varepsilon) \rightarrow a$ as $\varepsilon \rightarrow 0$.

For the proof of theorem 7 please see Corollary 1 of [27].

\section{Proof of Theorems}

\subsection{Proof of Theorem 1}

We shall apply Theorem 7 to the differential system (1.1). It can be written as system (2.1) taking

$$
\boldsymbol{x}=\left(\begin{array}{l}
x \\
y \\
u \\
v
\end{array}\right), t=t, F_{0}(t, \boldsymbol{x})=\left(\begin{array}{c}
-y+h_{1}(t) \\
x+h_{2}(t) \\
-v+h_{3}(t) \\
u+h_{4}(t)
\end{array}\right) \text { and } F_{1}(t, x)=\left(\begin{array}{c}
P_{1}(x, y, u, v) \\
P_{2}(x, y, u, v) \\
P_{3}(x, y, u, v) \\
P_{4}(x, y, u, v)
\end{array}\right)
$$

We shall study the periodic solutions of system (2.2) in our case the system $(1.1)_{\varepsilon=0}$.

By using

$$
\left(\begin{array}{l}
x(t) \\
y(t) \\
u(t) \\
v(t)
\end{array}\right)=e^{A t}\left(\begin{array}{l}
x_{0} \\
y_{0} \\
u_{0} \\
v_{0}
\end{array}\right)+\int_{0}^{t} e^{A(t-s)}\left(\begin{array}{l}
h_{1}(s) \\
h_{2}(s) \\
h_{3}(s) \\
h_{4}(s)
\end{array}\right) \mathrm{d} s
$$


we obtain

$$
\left(\begin{array}{l}
x(t) \\
y(t) \\
u(t) \\
v(t)
\end{array}\right)=\left(\begin{array}{l}
\cos (t) x_{0}-\sin (t) y_{0}+\int_{0}^{t}\left(\cos (t-s) h_{1}(s)-\sin (t-s) h_{2}(s)\right) \mathrm{d} s \\
\sin (t) x_{0}+\cos (t) y_{0}+\int_{0}^{t}\left(\sin (t-s) h_{1}(s)+\cos (t-s) h_{2}(s)\right) \mathrm{d} s \\
\cos (t) u_{0}-\sin (t) v_{0}+\int_{0}^{t}\left(\cos (t-s) h_{3}(s)-\sin (t-s) h_{4}(s)\right) \mathrm{d} s \\
\sin (t) u_{0}+\cos (t) v_{0}+\int_{0}^{t}\left(\sin (t-s) h_{3}(s)+\cos (t-s) h_{4}(s)\right) \mathrm{d} s
\end{array}\right),
$$

it can be written as

$$
\begin{aligned}
\left(\begin{array}{l}
x(t) \\
y(t) \\
u(t) \\
v(t)
\end{array}\right)= & \left(\begin{array}{c}
\cos (t) \\
\sin (t) \\
0 \\
0
\end{array}\right) x_{0}+\left(\begin{array}{c}
-\sin (t) \\
\cos (t) \\
0 \\
0
\end{array}\right) y_{0}+\left(\begin{array}{c}
0 \\
0 \\
\cos (t) \\
\sin (t)
\end{array}\right) u_{0}+\left(\begin{array}{c}
0 \\
0 \\
-\sin (t) \\
\cos (t)
\end{array}\right) v_{0} \\
& +\left(\begin{array}{l}
\int_{0}^{t}\left(\cos (t-s) h_{1}(s)-\sin (t-s) h_{2}(s)\right) \mathrm{d} s \\
\int_{0}^{t}\left(\sin (t-s) h_{1}(s)+\cos (t-s) h_{2}(s)\right) \mathrm{d} s \\
\int_{0}^{t}\left(\cos (t-s) h_{3}(s)-\sin (t-s) h_{4}(s)\right) \mathrm{d} s \\
\int_{0}^{t}\left(\sin (t-s) h_{3}(s)+\cos (t-s) h_{4}(s)\right) \mathrm{d} s
\end{array}\right)
\end{aligned}
$$

These solutions are $2 \pi$-periodic if and only if

$$
\left(\begin{array}{l}
x(2 \pi) \\
y(2 \pi) \\
u(2 \pi) \\
v(2 \pi)
\end{array}\right)=\left(\begin{array}{l}
x(0) \\
y(0) \\
u(0) \\
v(0)
\end{array}\right)
$$

We obtain the periodicity conditions given in the theorem 1 by (1.2).

The set of periodic solutions has dimension 4 . To look for the periodic solutions of our system (1.1) we must calculate the zeros $\mathbf{z}=\left(x_{0}, y_{0}, u_{0}, v_{0}\right)$ of the system $\mathcal{F}(\mathbf{z})=0$, where $\mathcal{F}(\mathbf{z})$ is given by (2.5). The fundamental matrix $\boldsymbol{M}(t)$ of the differential system (2.3) is

$$
\boldsymbol{M}(t)=\boldsymbol{M}_{z}(t)=\left(\begin{array}{cccc}
\cos (t) & -\sin (t) & 0 & 0 \\
\sin (t) & \cos (t) & 0 & 0 \\
0 & 0 & \cos (t) & -\sin (t) \\
0 & 0 & \sin (t) & \cos (t)
\end{array}\right) .
$$

Now computing the function $\mathcal{F}(\mathbf{z})$ we find the system

$$
\left\{\begin{array}{l}
\mathcal{F}_{1}\left(x_{0}, y_{0}, u_{0}, v_{0}\right)=0, \\
\mathcal{F}_{2}\left(x_{0}, y_{0}, u_{0}, v_{0}\right)=0, \\
\mathcal{F}_{3}\left(x_{0}, y_{0}, u_{0}, v_{0}\right)=0, \\
\mathcal{F}_{4}\left(x_{0}, y_{0}, u_{0}, v_{0}\right)=0,
\end{array}\right.
$$

where $\mathcal{F}_{1}, \mathcal{F}_{2}, \mathcal{F}_{3}$ and $\mathcal{F}_{4}$ have been defined in the statement of Theorem 1. The zeros $\left(x_{0}^{*}, y_{0}^{*}, u_{0}^{*}, v_{0}^{*}\right)$ of the system (3.2) with respect to the variables $x_{0}$, $y_{0}, u_{0}$ and $v_{0}$ provide periodic solutions of system (1.1) with $\varepsilon \neq 0$ being sufficiently small if they are simple, i.e. if 


$$
\operatorname{det}\left(\left.\frac{\partial\left(\mathcal{F}_{1}, \mathcal{F}_{2}, \mathcal{F}_{3}, \mathcal{F}_{4}\right)}{\partial\left(x_{0}, y_{0}, u_{0}, v_{0}\right)}\right|_{\left(x_{0}, y_{0}, u_{0}, v_{0}\right)=\left(x_{0}^{*}, y_{0}^{*}, u_{0}^{*}, v_{0}^{*}\right)}\right) \neq 0 .
$$

For simple zero $\left(x_{0}^{*}, y_{0}^{*}, u_{0}^{*}, v_{0}^{*}\right)$ of system (3.2) we obtain a $2 \pi$-periodic solution $\left(\begin{array}{l}x(t, \varepsilon) \\ y(t, \varepsilon) \\ u(t, \varepsilon) \\ v(t, \varepsilon)\end{array}\right)$ of the differential system (1.1), for $\varepsilon \neq 0$ being sufficiently small which tends to the periodic solution given in the statement of theorem 1 of the differential system $(1.1)_{\varepsilon=0}$ when $\varepsilon \rightarrow 0$.

This completes the proof of Theorem 1 .

\subsection{Proof of Theorem 2}

We shall apply Theorem 6 to the differential system (1.1). It can be written as system (2.1) taking

$$
\boldsymbol{x}=\left(\begin{array}{l}
x \\
y \\
u \\
v
\end{array}\right), t=t, F_{0}(t, \boldsymbol{x})=\left(\begin{array}{c}
-y+h_{1}(t) \\
x+h_{2}(t) \\
-v+h_{3}(t) \\
u+h_{4}(t)
\end{array}\right) \text { and } F_{1}(t, \boldsymbol{x})=\left(\begin{array}{c}
P_{1}(x, y, u, v) \\
P_{2}(x, y, u, v) \\
P_{3}(x, y, u, v) \\
P_{4}(x, y, u, v)
\end{array}\right) \text {. }
$$

We shall study the periodic solutions of system (2.2) in our case the system $(1.1)_{\varepsilon=0}$.

$$
\text { By using }
$$

$$
\left(\begin{array}{l}
x(t) \\
y(t) \\
u(t) \\
v(t)
\end{array}\right)=e^{A t}\left(\begin{array}{l}
x_{0} \\
y_{0} \\
u_{0} \\
v_{0}
\end{array}\right)+\int_{0}^{t} e^{A(t-s)}\left(\begin{array}{l}
h_{1}(s) \\
h_{2}(s) \\
h_{3}(s) \\
h_{4}(s)
\end{array}\right) \mathrm{d} s
$$

we obtain

$$
\left(\begin{array}{c}
x(t) \\
y(t) \\
u(t) \\
v(t)
\end{array}\right)=\left(\begin{array}{c}
\cos (t) x_{0}-\sin (t) y_{0}+\int_{0}^{t}\left(\cos (t-s) h_{1}(s)-\sin (t-s) h_{2}(s)\right) \mathrm{d} s \\
\sin (t) x_{0}+\cos (t) y_{0}+\int_{0}^{t}\left(\sin (t-s) h_{1}(s)+\cos (t-s) h_{2}(s)\right) \mathrm{d} s \\
e^{\lambda t} u_{0}+\int_{0}^{t} e^{\lambda(t-s)} h_{3}(s) \mathrm{d} s \\
e^{\mu t} v_{0}+\int_{0}^{t} e^{\mu(t-s)} h_{4}(s) \mathrm{d} s
\end{array}\right),
$$

it can be written as

$$
\begin{aligned}
\left(\begin{array}{l}
x(t) \\
y(t) \\
u(t) \\
v(t)
\end{array}\right)= & \left(\begin{array}{c}
\cos (t) \\
\sin (t) \\
0 \\
0
\end{array}\right) x_{0}+\left(\begin{array}{c}
-\sin (t) \\
\cos (t) \\
0 \\
0
\end{array}\right) y_{0}+\left(\begin{array}{c}
0 \\
0 \\
e^{\lambda t} \\
0
\end{array}\right) u_{0} \\
& +\left(\begin{array}{c}
0 \\
0 \\
0 \\
e^{\mu t}
\end{array}\right) v_{0}+\left(\begin{array}{c}
\int_{0}^{t}\left(\cos (t-s) h_{1}(s)-\sin (t-s) h_{2}(s)\right) \mathrm{d} s \\
\int_{0}^{t}\left(\sin (t-s) h_{1}(s)+\cos (t-s) h_{2}(s)\right) \mathrm{d} s \\
\int_{0}^{t} e^{\lambda(t-s)} h_{3}(s) \mathrm{d} s \\
\int_{0}^{t} e^{\mu(t-s)} h_{4}(s) \mathrm{d} s
\end{array}\right)
\end{aligned}
$$


These solutions are $2 \pi$-periodic if and only if

$$
\left(\begin{array}{l}
x(2 \pi) \\
y(2 \pi) \\
u(2 \pi) \\
v(2 \pi)
\end{array}\right)=\left(\begin{array}{l}
x(0) \\
y(0) \\
u(0) \\
v(0)
\end{array}\right)
$$

We obtain the periodicity conditions given in the theorem 2 by (1.3). Since $u_{0}$ and $v_{0}$ are now fixed then the set of periodic solutions has dimension 2. To look for the periodic solutions of our system (1.1) we must calculate the zeros $\mathbf{z}=\left(x_{0}, y_{0}\right)$ of the system $\mathcal{F}(\mathbf{z})=0$, where $\mathcal{F}(\mathbf{z})$ is given by (2.4). The fundamental matrix $\boldsymbol{M}(t)$ of the differential system (2.3) is

$$
\boldsymbol{M}(t)=\boldsymbol{M}_{z}(t)=\left(\begin{array}{cccc}
\cos (t) & -\sin (t) & 0 & 0 \\
\sin (t) & \cos (t) & 0 & 0 \\
0 & 0 & e^{\lambda t} & 0 \\
0 & 0 & 0 & e^{\mu t}
\end{array}\right) .
$$

Now computing the function $\mathcal{F}(\mathbf{z})$ we find the system

$$
\left\{\begin{array}{l}
\mathcal{F}_{1}\left(x_{0}, y_{0}\right)=0 \\
\mathcal{F}_{2}\left(x_{0}, y_{0}\right)=0
\end{array}\right.
$$

where $\mathcal{F}_{1}$ and $\mathcal{F}_{2}$ have been defined in the statement of Theorem 2 .

The zeros $\left(x_{0}^{*}, y_{0}^{*}\right)$ of the system (3.4) with respect to the variables $x_{0}, y_{0}$ provide periodic solutions of system (1.1) with $\varepsilon \neq 0$ being sufficiently small if they are simple, i.e. if

$$
\operatorname{det}\left(\left.\frac{\partial\left(\mathcal{F}_{1}, \mathcal{F}_{2}\right)}{\partial\left(x_{0}, y_{0}\right)}\right|_{\left(x_{0}, y_{0}\right)=\left(x_{0}^{*}, y_{0}^{*}\right)}\right) \neq 0 .
$$

For simple zeros $\left(x_{0}^{*}, y_{0}^{*}\right)$ of system (3.2) we obtain a $2 \pi$-periodic solution $\left(\begin{array}{l}x(t, \varepsilon) \\ y(t, \varepsilon) \\ u(t, \varepsilon) \\ v(t, \varepsilon)\end{array}\right)$ of the differential system (1.1), for $\varepsilon \neq 0$ being sufficiently small which tends to the periodic solution given in the statement of theorem 2 of the differential system $(1.1)_{\varepsilon=0}$ when $\varepsilon \rightarrow 0$.

This completes the proof of Theorem 2 .

\subsection{Proof of Theorem 3}

We shall apply Theorem 6 to the differential system (1.1). It can be written as system (2.1) taking

$$
\boldsymbol{x}=\left(\begin{array}{l}
x \\
y \\
u \\
v
\end{array}\right), t=t, F_{0}(t, \boldsymbol{x})=\left(\begin{array}{c}
-y+h_{1}(t) \\
x+h_{2}(t) \\
h_{3}(t) \\
\mu v+h_{4}(t)
\end{array}\right) \text { and } F_{1}(t, \boldsymbol{x})=\left(\begin{array}{c}
P_{1}(x, y, u, v) \\
P_{2}(x, y, u, v) \\
P_{3}(x, y, u, v) \\
P_{4}(x, y, u, v)
\end{array}\right) \text {. }
$$


We shall study the periodic solutions of system (2.2) in our case the system $(1.1)_{\varepsilon=0}$.

By using

$$
\left(\begin{array}{l}
x(t) \\
y(t) \\
u(t) \\
v(t)
\end{array}\right)=e^{A t}\left(\begin{array}{l}
x_{0} \\
y_{0} \\
u_{0} \\
v_{0}
\end{array}\right)+\int_{0}^{t} e^{A(t-s)}\left(\begin{array}{l}
h_{1}(s) \\
h_{2}(s) \\
h_{3}(s) \\
h_{4}(s)
\end{array}\right) \mathrm{d} s,
$$

we obtain

$$
\left(\begin{array}{c}
x(t) \\
y(t) \\
u(t) \\
v(t)
\end{array}\right)=\left(\begin{array}{c}
\cos (t) x_{0}-\sin (t) y_{0}+\int_{0}^{t}\left(\cos (t-s) h_{1}(s)-\sin (t-s) h_{2}(s)\right) \mathrm{d} s \\
\sin (t) x_{0}+\cos (t) y_{0}+\int_{0}^{t}\left(\sin (t-s) h_{1}(s)+\cos (t-s) h_{2}(s)\right) \mathrm{d} s \\
u_{0}+\int_{0}^{t} h_{3}(s) \mathrm{d} s \\
e^{\mu t} v_{0}+\int_{0}^{t} e^{\mu(t-s)} h_{4}(s) \mathrm{d} s
\end{array}\right),
$$

it can be written as

$$
\begin{aligned}
\left(\begin{array}{l}
x(t) \\
y(t) \\
u(t) \\
v(t)
\end{array}\right)=\left(\begin{array}{c}
\cos (t) \\
\sin (t) \\
0 \\
0
\end{array}\right) x_{0}+\left(\begin{array}{c}
-\sin (t) \\
\cos (t) \\
0 \\
0
\end{array}\right) y_{0}+\left(\begin{array}{l}
0 \\
0 \\
1 \\
0
\end{array}\right) u_{0} \\
+\left(\begin{array}{c}
0 \\
0 \\
0 \\
e^{\mu t}
\end{array}\right) v_{0}+\left(\begin{array}{c}
\int_{0}^{t}\left(\cos (t-s) h_{1}(s)-\sin (t-s) h_{2}(s)\right) \mathrm{d} s \\
\int_{0}^{t}\left(\sin (t-s) h_{1}(s)+\cos (t-s) h_{2}(s)\right) \mathrm{d} s \\
\int_{0}^{t} h_{3}(s) \mathrm{d} s \\
\int_{0}^{t} e^{\mu(t-s)} h_{4}(s) \mathrm{d} s
\end{array}\right)
\end{aligned}
$$

These solutions are $2 \pi$-periodic if and only if

$$
\left(\begin{array}{l}
x(2 \pi) \\
y(2 \pi) \\
u(2 \pi) \\
v(2 \pi)
\end{array}\right)=\left(\begin{array}{l}
x(0) \\
y(0) \\
u(0) \\
v(0)
\end{array}\right)
$$

We obtain the periodicity conditions given in the theorem 3 by (1.4). Since $v_{0}$ is now fixed then the set of periodic solutions has dimension 3 . To look for the periodic solutions of our system (1.1) we must calculate the zeros $\mathbf{z}=\left(x_{0}, y_{0}, u_{0}\right)$ of the system $\mathcal{F}(\mathbf{z})=0$, where $\mathcal{F}(\mathbf{z})$ is given by (2.4). The fundamental matrix $\boldsymbol{M}(t)$ of the differential system (2.3) is

$$
\boldsymbol{M}(t)=\boldsymbol{M}_{z}(t)=\left(\begin{array}{cccc}
\cos (t) & -\sin (t) & 0 & 0 \\
\sin (t) & \cos (t) & 0 & 0 \\
0 & 0 & 1 & 0 \\
0 & 0 & 0 & e^{\mu t}
\end{array}\right) .
$$

Now computing the function $\mathcal{F}(\mathbf{z})$ we find the system 


$$
\left\{\begin{array}{l}
\mathcal{F}_{1}\left(x_{0}, y_{0}, u_{0}\right)=0, \\
\mathcal{F}_{2}\left(x_{0}, y_{0}, u_{0}\right)=0, \\
\mathcal{F}_{3}\left(x_{0}, y_{0}, u_{0}\right)=0,
\end{array}\right.
$$

where $\mathcal{F}_{1}, \mathcal{F}_{2}$ and $\mathcal{F}_{3}$ have been defined in the statement of Theorem 3.

The zeros $\left(x_{0}^{*}, y_{0}^{*}, u_{0}^{*}\right)$ of the system (3.5) with respect to the variables $x_{0}, y_{0}$ and $u_{0}$ provide periodic solutions of system (1.1) with $\varepsilon \neq 0$ being sufficiently small if they are simple, i.e. if

$$
\operatorname{det}\left(\left.\frac{\partial\left(\mathcal{F}_{1}, \mathcal{F}_{2}, \mathcal{F}_{3}\right)}{\partial\left(x_{0}, y_{0}, u_{0}\right)}\right|_{\left(x_{0}, y_{0}, u_{0}\right)=\left(x_{0}^{*}, y_{0}^{*}, u_{0}^{*}\right)}\right) \neq 0 .
$$

For simple zeros $\left(x_{0}^{*}, y_{0}^{*}, u_{0}^{*}\right)$ of system (3.5) we obtain a $2 \pi$-periodic solution $\left(\begin{array}{l}x(t, \varepsilon) \\ y(t, \varepsilon) \\ u(t, \varepsilon) \\ v(t, \varepsilon)\end{array}\right)$ of the differential system (1.1), for $\varepsilon \neq 0$ being sufficiently small which tends to the periodic solution given in the statement of theorem 3 of the differential system $(1.1)_{\varepsilon=0}$ when $\varepsilon \rightarrow 0$.

This completes the proof of Theorem 3 .

\subsection{Proof of Theorem 4}

We shall apply Theorem 7 to the differential system (1.1). It can be written as system (2.1) taking

$$
\boldsymbol{x}=\left(\begin{array}{c}
x \\
y \\
u \\
v
\end{array}\right), t=t, F_{0}(t, \boldsymbol{x})=\left(\begin{array}{c}
-y+h_{1}(t) \\
x+h_{2}(t) \\
h_{3}(t) \\
h_{4}(t)
\end{array}\right) \text { and } F_{1}(t, \boldsymbol{x})=\left(\begin{array}{c}
P_{1}(x, y, u, v) \\
P_{2}(x, y, u, v) \\
P_{3}(x, y, u, v) \\
P_{4}(x, y, u, v)
\end{array}\right) .
$$

We shall study the periodic solutions of system (2.2) in our case the system $(1.1)_{\varepsilon=0}$.

By using

$$
\left(\begin{array}{l}
x(t) \\
y(t) \\
u(t) \\
v(t)
\end{array}\right)=e^{A t}\left(\begin{array}{l}
x_{0} \\
y_{0} \\
u_{0} \\
v_{0}
\end{array}\right)+\int_{0}^{t} e^{A(t-s)}\left(\begin{array}{l}
h_{1}(s) \\
h_{2}(s) \\
h_{3}(s) \\
h_{4}(s)
\end{array}\right) \mathrm{d} s
$$

we obtain

$$
\left(\begin{array}{l}
x(t) \\
y(t) \\
u(t) \\
v(t)
\end{array}\right)=\left(\begin{array}{c}
\cos (t) x_{0}-\sin (t) y_{0}+\int_{0}^{t}\left(\cos (t-s) h_{1}(s)-\sin (t-s) h_{2}(s)\right) \mathrm{d} s \\
\sin (t) x_{0}+\cos (t) y_{0}+\int_{0}^{t}\left(\sin (t-s) h_{1}(s)+\cos (t-s) h_{2}(s)\right) \mathrm{d} s \\
u_{0}+\int_{0}^{t} h_{3}(s) \mathrm{d} s \\
v_{0}+\int_{0}^{t} h_{4}(s) \mathrm{d} s
\end{array}\right),
$$

it can be written as 


$$
\begin{aligned}
\left(\begin{array}{l}
x(t) \\
y(t) \\
u(t) \\
v(t)
\end{array}\right)=\left(\begin{array}{c}
\cos (t) \\
\sin (t) \\
0 \\
0
\end{array}\right) x_{0}+\left(\begin{array}{c}
-\sin (t) \\
\cos (t) \\
0 \\
0
\end{array}\right) y_{0}+\left(\begin{array}{l}
0 \\
0 \\
1 \\
0
\end{array}\right) u_{0} \\
+\left(\begin{array}{l}
0 \\
0 \\
0 \\
1
\end{array}\right) v_{0}+\left(\begin{array}{c}
\int_{0}^{t}\left(\cos (t-s) h_{1}(s)-\sin (t-s) h_{2}(s)\right) \mathrm{d} s \\
\int_{0}^{t}\left(\sin (t-s) h_{1}(s)+\cos (t-s) h_{2}(s)\right) \mathrm{d} s \\
u_{0}+\int_{0}^{t} h_{3}(s) \mathrm{d} s \\
v_{0}+\int_{0}^{t} h_{4}(s) \mathrm{d} s
\end{array}\right)
\end{aligned}
$$

These solutions are $2 \pi$-periodic if and only if

$$
\left(\begin{array}{l}
x(2 \pi) \\
y(2 \pi) \\
u(2 \pi) \\
v(2 \pi)
\end{array}\right)=\left(\begin{array}{c}
x(0) \\
y(0) \\
u(0) \\
v(0)
\end{array}\right)
$$

We obtain the periodicity conditions given in the theorem 4 by (1.5).

The set of periodic solutions has dimension 4 . To look for the periodic solutions of our system (1.1) we must calculate the zeros $\mathbf{z}=\left(x_{0}, y_{0}, u_{0}, v_{0}\right)$ of the system $\mathcal{F}(\mathbf{z})=0$, where $\mathcal{F}(\mathbf{z})$ is given by (2.5). The fundamental matrix $\boldsymbol{M}(t)$ of the differential system (2.3) is

$$
\boldsymbol{M}(t)=\boldsymbol{M}_{z}(t)=\left(\begin{array}{cccc}
\cos (t) & -\sin (t) & 0 & 0 \\
\sin (t) & \cos (t) & 0 & 0 \\
0 & 0 & 1 & 0 \\
0 & 0 & 0 & 1
\end{array}\right) .
$$

Now computing the function $\mathcal{F}(\mathbf{z})$ we find the system

$$
\left\{\begin{array}{l}
\mathcal{F}_{1}\left(x_{0}, y_{0}, u_{0}, v_{0}\right)=0 \\
\mathcal{F}_{2}\left(x_{0}, y_{0}, u_{0}, v_{0}\right)=0 \\
\mathcal{F}_{3}\left(x_{0}, y_{0}, u_{0}, v_{0}\right)=0 \\
\mathcal{F}_{4}\left(x_{0}, y_{0}, u_{0}, v_{0}\right)=0
\end{array}\right.
$$

where $\mathcal{F}_{1}, \mathcal{F}_{2}, \mathcal{F}_{3}$ and $\mathcal{F}_{4}$ have been defined in the statement of Theorem 4.

The zeros $\left(x_{0}^{*}, y_{0}^{*}, u_{0}^{*}, v_{0}^{*}\right)$ of the system (3.8) with respect to the variables $x_{0}$, $y_{0}, u_{0}$ and $v_{0}$ provide periodic solutions of system (1.1) and $\varepsilon \neq 0$ being sufficiently small if they are simple, i.e. if

$$
\operatorname{det}\left(\left.\frac{\partial\left(\mathcal{F}_{1}, \mathcal{F}_{2}, \mathcal{F}_{3}, \mathcal{F}_{4}\right)}{\partial\left(x_{0}, y_{0}, u_{0}, v_{0}\right)}\right|_{\left(x_{0}, y_{0}, u_{0}, v_{0}\right)=\left(x_{0}^{*}, y_{0}^{*}, u_{0}^{*}, v_{0}^{*}\right)}\right) \neq 0 .
$$

For simple zeros $\left(x_{0}^{*}, y_{0}^{*}, u_{0}^{*}, v_{0}^{*}\right)$ of system (3.8) we obtain a $2 \pi$-periodic solution $\left(\begin{array}{l}x(t, \varepsilon) \\ y(t, \varepsilon) \\ u(t, \varepsilon) \\ v(t, \varepsilon)\end{array}\right)$ of the differential system (1.1), for $\varepsilon \neq 0$ being sufficiently 
small which tends to the periodic solution given in the statement of theorem 4 of the differential system $(1.1)_{\varepsilon=0}$ when $\varepsilon \rightarrow 0$.

This completes the proof of Theorem 4 .

\subsection{Proof of Theorem 5}

We shall apply Theorem 6 to the differential system (1.1). It can be written as system (2.1) taking

$$
\boldsymbol{x}=\left(\begin{array}{l}
x \\
y \\
u \\
v
\end{array}\right), t=t, F_{0}(t, \boldsymbol{x})=\left(\begin{array}{c}
-y+h_{1}(t) \\
x+h_{2}(t) \\
\lambda u+v+h_{3}(t) \\
\lambda v+h_{4}(t)
\end{array}\right) \text { and } F_{1}(t, \boldsymbol{x})=\left(\begin{array}{c}
P_{1}(x, y, u, v) \\
P_{2}(x, y, u, v) \\
P_{3}(x, y, u, v) \\
P_{4}(x, y, u, v)
\end{array}\right) \text {. }
$$

We shall study the periodic solutions of system (2.2) in our case the system $(1.1)_{\varepsilon=0}$.

By using

$$
\left(\begin{array}{l}
x(t) \\
y(t) \\
u(t) \\
v(t)
\end{array}\right)=e^{A t}\left(\begin{array}{l}
x_{0} \\
y_{0} \\
u_{0} \\
v_{0}
\end{array}\right)+\int_{0}^{t} e^{A(t-s)}\left(\begin{array}{l}
h_{1}(s) \\
h_{2}(s) \\
h_{3}(s) \\
h_{4}(s)
\end{array}\right) \mathrm{d} s
$$

we obtain

$$
\left(\begin{array}{l}
x(t) \\
y(t) \\
u(t) \\
v(t)
\end{array}\right)=\left(\begin{array}{c}
\cos (t) x_{0}-\sin (t) y_{0}+\int_{0}^{t}\left(\cos (t-s) h_{1}(s)-\sin (t-s) h_{2}(s)\right) \mathrm{d} s \\
\sin (t) x_{0}+\cos (t) y_{0}+\int_{0}^{t}\left(\sin (t-s) h_{1}(s)+\cos (t-s) h_{2}(s)\right) \mathrm{d} s \\
e^{\lambda t} u_{0}+t e^{\lambda t} v_{0}+\int_{0}^{t} e^{\lambda(t-s)}\left(h_{3}(s)+(t-s) h_{4}(s)\right) \mathrm{d} s \\
e^{\lambda t} v_{0}+\int_{0}^{t} e^{\lambda(t-s)} h_{4}(s) \mathrm{d} s
\end{array}\right),
$$

it can be written as

$$
\begin{aligned}
\left(\begin{array}{l}
x(t) \\
y(t) \\
u(t) \\
v(t)
\end{array}\right) & \left(\begin{array}{c}
\cos (t) \\
\sin (t) \\
0 \\
0
\end{array}\right) x_{0}+\left(\begin{array}{c}
-\sin (t) \\
\cos (t) \\
0 \\
0
\end{array}\right) y_{0}+\left(\begin{array}{c}
0 \\
0 \\
e^{\lambda t} \\
0
\end{array}\right) u_{0} \\
& +\left(\begin{array}{c}
0 \\
0 \\
t e^{\lambda t} \\
e^{\lambda t}
\end{array}\right) v_{0}+\left(\begin{array}{c}
\int_{0}^{t}\left(\cos (t-s) h_{1}(s)-\sin (t-s) h_{2}(s)\right) \mathrm{d} s \\
\int_{0}^{t}\left(\sin (t-s) h_{1}(s)+\cos (t-s) h_{2}(s)\right) \mathrm{d} s \\
\int_{0}^{t} e^{\lambda(t-s)}\left(h_{3}(s)+(t-s) h_{4}(s)\right) \mathrm{d} s \\
\int_{0}^{t} e^{\lambda(t-s)} h_{4}(s) \mathrm{d} s
\end{array}\right)
\end{aligned}
$$

These solutions are $2 \pi$-periodic if and only if

$$
\left(\begin{array}{l}
x(2 \pi) \\
y(2 \pi) \\
u(2 \pi) \\
v(2 \pi)
\end{array}\right)=\left(\begin{array}{l}
x(0) \\
y(0) \\
u(0) \\
v(0)
\end{array}\right)
$$


We obtain the periodicity conditions given in the theorem 5 by (1.6). Since $u_{0}$ and $v_{0}$ are now fixed then the set of periodic solutions has dimension 2. To look for the periodic solutions of our system (1.1) we must calculate the zeros $\mathbf{z}=\left(x_{0}, y_{0}\right)$ of the system $\mathcal{F}(\mathbf{z})=0$, where $\mathcal{F}(\mathbf{z})$ is given by (2.4). The fundamental matrix $\boldsymbol{M}(t)$ of the differential system (2.3) is

$$
\boldsymbol{M}(t)=\boldsymbol{M}_{z}(t)=\left(\begin{array}{cccc}
\cos (t) & -\sin (t) & 0 & 0 \\
\sin (t) & \cos (t) & 0 & 0 \\
0 & 0 & e^{\lambda t} & t e^{\lambda t} \\
0 & 0 & 0 & e^{\lambda t}
\end{array}\right) .
$$

Now computing the function $\mathcal{F}(\mathbf{z})$ we find the system

$$
\left\{\begin{array}{l}
\mathcal{F}_{1}\left(x_{0}, y_{0}\right)=0, \\
\mathcal{F}_{2}\left(x_{0}, y_{0}\right)=0,
\end{array}\right.
$$

where $\mathcal{F}_{1}$ and $\mathcal{F}_{2}$ have been defined in the statement of Theorem 5. The ze$\operatorname{ros}\left(x_{0}^{*}, y_{0}^{*}\right)$ of the system (3.10) with respect to the variables $x_{0}, y_{0}$ provide periodic solutions of system (1.1) and $\varepsilon \neq 0$ being sufficiently small if they are simple, i.e. if

$$
\operatorname{det}\left(\left.\frac{\partial\left(\mathcal{F}_{1}, \mathcal{F}_{2}\right)}{\partial\left(x_{0}, y_{0}\right)}\right|_{\left(x_{0}, y_{0}\right)=\left(x_{0}^{*}, y_{0}^{*}\right)}\right) \neq 0 .
$$

For simple zeros $\left(x_{0}^{*}, y_{0}^{*}\right)$ of system (3.10) we obtain a $2 \pi$-periodic solution $\left(\begin{array}{l}x(t, \varepsilon) \\ y(t, \varepsilon) \\ u(t, \varepsilon) \\ v(t, \varepsilon)\end{array}\right)$ of the differential system (1.1), for $\varepsilon \neq 0$ being sufficiently small which tends to the periodic solution given in the statement of theorem 5 of the differential system $(1.1)_{\varepsilon=0}$ when $\varepsilon \rightarrow 0$.

This completes the proof of Theorem 5 .

\section{Applications}

\subsection{Application of Theorem 1}

Consider the differential system (1) where

$$
\begin{gathered}
\boldsymbol{A}=\left(\begin{array}{cccc}
0 & -1 & 0 & 0 \\
1 & 0 & 0 & 0 \\
0 & 0 & 0 & -1 \\
0 & 0 & 1 & 0
\end{array}\right), \\
\left(\begin{array}{l}
h_{1}(t) \\
h_{2}(t) \\
h_{3}(t) \\
h_{4}(t)
\end{array}\right)=\left(\begin{array}{c}
\sin (t) \\
\cos (t) \\
\sin (t) \\
\cos (t)
\end{array}\right)
\end{gathered}
$$

and 


$$
\left(\begin{array}{c}
P_{1}(x, y, u, v) \\
P_{2}(x, y, u, v) \\
P_{3}(x, y, u, v) \\
P_{4}(x, y, u, v)
\end{array}\right)=\left(\begin{array}{c}
x+y-y x^{2} \\
x+y-x y^{2} \\
x+y+u+v \\
x+y+u+v
\end{array}\right)
$$

We can easily verify conditions (1.2)

$$
\begin{gathered}
\int_{0}^{2 \pi}(\cos (s) \sin (s)-\sin (s) \cos (s)) \mathrm{d} s=0, \\
\int_{0}^{2 \pi}\left(-\sin ^{2}(s)+\cos ^{2}(s)\right) \mathrm{d} s=0, \\
\int_{0}^{2 \pi}(\cos (s) \sin (s)-\sin (s) \cos (s)) \mathrm{d} s=0, \\
\int_{0}^{2 \pi}\left(-\sin ^{2}(s)+\cos ^{2}(s)\right) \mathrm{d} s=0,
\end{gathered}
$$

Computing the functions $\mathcal{F}_{1}, \mathcal{F}_{2}, \mathcal{F}_{3}$ and $\mathcal{F}_{4}$ we find

$$
\begin{gathered}
\mathcal{F}_{1}\left(x_{0}, y_{0}, u_{0}, v_{0}\right)=\frac{1}{2}+x_{0}+\frac{3}{8} y_{0}+\frac{3}{4} x_{0} y_{0}, \\
\mathcal{F}_{2}\left(x_{0}, y_{0}, u_{0}, v_{0}\right)=-\frac{1}{2}-\frac{1}{8} x_{0}-\frac{1}{8} x_{0}^{2}+y_{0}+\frac{5}{8} y_{0}^{2}, \\
\mathcal{F}_{3}\left(x_{0}, y_{0}, u_{0}, v_{0}\right)=x_{0}+u_{0}+1, \\
\mathcal{F}_{4}\left(x_{0}, y_{0}, u_{0}, v_{0}\right)=y_{0}+v_{0}-1 .
\end{gathered}
$$

The stability of the periodic solutions associated to a simple zero of $\mathcal{F}$ is controlled by the eigenvalues of the jacobian matrix.

The system $\mathcal{F}_{1}=\mathcal{F}_{2}=\mathcal{F}_{3}=\mathcal{F}_{4}=0$ has four solutions $\left(x_{0}^{*}, y_{0}^{*}, u_{0}^{*}, v_{0}^{*}\right)$ given by

$$
\begin{aligned}
& \left(-\frac{1}{2},-\frac{4}{5}+\frac{\sqrt{139}}{10},-\frac{1}{2}, \frac{9}{5}-\frac{\sqrt{139}}{10}\right),\left(-\frac{1}{2},-\frac{4}{5}-\frac{\sqrt{139}}{10},-\frac{1}{2}, \frac{9}{5}-\frac{\sqrt{139}}{10}\right), \\
& \left(-\frac{1}{2},-\frac{4}{5}+\frac{\sqrt{139}}{10},-\frac{1}{2}, \frac{9}{5}+\frac{\sqrt{139}}{10}\right),\left(-\frac{1}{2},-\frac{4}{5}-\frac{\sqrt{139}}{10},-\frac{1}{2}, \frac{9}{5}+\frac{\sqrt{139}}{10}\right)
\end{aligned}
$$

and the eigenvalues of the jacobian matrix of $\left(\begin{array}{l}\mathcal{F}_{1}\left(x_{0}, y_{0}, u_{0}, v_{0}\right) \\ \mathcal{F}_{2}\left(x_{0}, y_{0}, u_{0}, v_{0}\right) \\ \mathcal{F}_{3}\left(x_{0}, y_{0}, u_{0}, v_{0}\right) \\ \mathcal{F}_{4}\left(x_{0}, y_{0}, u_{0}, v_{0}\right)\end{array}\right)$ at these solu-

tions are $\left(\begin{array}{c}\frac{\sqrt{139}}{8} \\ \frac{2}{5}+\frac{3 \sqrt{139}}{40} \\ 1 \\ 1\end{array}\right),\left(\begin{array}{c}\frac{\sqrt{139}}{8} \\ \frac{2}{5}+\frac{3 \sqrt{139}}{40} \\ 1 \\ 1\end{array}\right),\left(\begin{array}{c}\frac{2}{5}-\frac{3 \sqrt{139}}{40} \\ \frac{-\sqrt{139}}{8} \\ 1 \\ 1\end{array}\right)$ and $\left(\begin{array}{c}\frac{2}{5}-\frac{3 \sqrt{139}}{40} \\ \frac{-\sqrt{139}}{8} \\ 1 \\ 1\end{array}\right)$, which

have all at least two positive real parts. Since

$$
\operatorname{det}\left(\left.\frac{\partial\left(\mathcal{F}_{1}, \mathcal{F}_{2}, \mathcal{F}_{3}, \mathcal{F}_{4}\right)}{\partial\left(x_{0}, y_{0}, u_{0}, v_{0}\right)}\right|_{\left(x_{0}, y_{0}, u_{0}, v_{0}\right)=\left(x_{0}^{*}, y_{0}^{*}, u_{0}^{*}, v_{0}^{*}\right)}\right)
$$

at these four solutions $\left(x_{0}^{*}, y_{0}^{*}, u_{0}^{*}, v_{0}^{*}\right)$ is $1,89,0,71,1,89,0,71$, respectively, then 
the differential system (1.1) has four periodic unstable solutions $\left(\begin{array}{l}x_{k}(t, \varepsilon) \\ y_{k}(t, \varepsilon) \\ u_{k}(t, \varepsilon) \\ v_{k}(t, \varepsilon)\end{array}\right)$ with $k=1,2,3,4$, tending to the unstable periodic solutions $\left(\begin{array}{l}x_{k}(t) \\ y_{k}(t) \\ u_{k}(t) \\ v_{k}(t)\end{array}\right)$ where

$$
\begin{aligned}
& \left(\begin{array}{l}
x_{1}(t) \\
y_{1}(t) \\
u_{1}(t) \\
v_{1}(t)
\end{array}\right)=\left(\begin{array}{c}
-\frac{1}{2} \cos (t)+\left(\frac{4}{5}-\frac{\sqrt{139}}{10}\right) \sin (t) \\
\frac{1}{2} \sin (t)+\left(-\frac{4}{5}+\frac{\sqrt{139}}{10}\right) \cos (t) \\
-\frac{1}{2} \cos (t)-\left(\frac{9}{5}+\frac{\sqrt{139}}{10}\right) \sin (t) \\
\frac{1}{2} \sin (t)+\left(\frac{9}{5}+\frac{\sqrt{139}}{10}\right) \cos (t)
\end{array}\right) \\
& \left(\begin{array}{l}
x_{2}(t) \\
y_{2}(t) \\
u_{2}(t) \\
v_{2}(t)
\end{array}\right)=\left(\begin{array}{c}
-\frac{1}{2} \cos (t)+\left(\frac{4}{5}-\frac{\sqrt{139}}{10}\right) \sin (t) \\
\frac{1}{2} \sin (t)+\left(-\frac{4}{5}+\frac{\sqrt{139}}{10}\right) \cos (t) \\
-\frac{1}{2} \cos (t)-\left(\frac{9}{5}-\frac{\sqrt{139}}{10}\right) \sin (t) \\
\frac{1}{2} \sin (t)+\left(\frac{9}{5}-\frac{\sqrt{139}}{10}\right) \cos (t)
\end{array}\right) \\
& \left(\begin{array}{l}
x_{3}(t) \\
y_{3}(t) \\
u_{3}(t) \\
v_{3}(t)
\end{array}\right)=\left(\begin{array}{c}
-\frac{1}{2} \cos (t)+\left(\frac{4}{5}+\frac{\sqrt{139}}{10}\right) \sin (t) \\
\frac{1}{2} \sin (t)-\left(\frac{4}{5}+\frac{\sqrt{139}}{10}\right) \cos (t) \\
-\frac{1}{2} \cos (t)-\left(\frac{9}{5}+\frac{\sqrt{139}}{10}\right) \sin (t) \\
\frac{1}{2} \sin (t)+\left(\frac{9}{5}+\frac{\sqrt{139}}{10}\right) \cos (t)
\end{array}\right) \\
& \left(\begin{array}{l}
x_{4}(t) \\
y_{4}(t) \\
u_{4}(t) \\
v_{4}(t)
\end{array}\right)=\left(\begin{array}{l}
-\frac{1}{2} \cos (t)+\left(\frac{4}{5}+\frac{\sqrt{139}}{10}\right) \sin (t) \\
\frac{1}{2} \sin (t)-\left(\frac{4}{5}+\frac{\sqrt{139}}{10}\right) \cos (t) \\
-\frac{1}{2} \cos (t)-\left(\frac{9}{5}-\frac{\sqrt{139}}{10}\right) \sin (t) \\
\frac{1}{2} \sin (t)+\left(\frac{9}{5}-\frac{\sqrt{139}}{10}\right) \cos (t)
\end{array}\right)
\end{aligned}
$$


of the differential system

$$
\begin{gathered}
\dot{x}=-y+\sin (t), \\
\dot{y}=x+\cos (t), \\
\dot{u}=-v+\sin (t), \\
\dot{v}=u+\cos (t)
\end{gathered}
$$

when $\varepsilon \rightarrow 0$.

\subsection{Application of Theorem 2}

Consider the differential system (1.1) where

$$
\begin{gathered}
\boldsymbol{A}=\left(\begin{array}{cccc}
0 & -1 & 0 & 0 \\
1 & 0 & 0 & 0 \\
0 & 0 & 3 & 0 \\
0 & 0 & 0 & 5
\end{array}\right), \\
\left(\begin{array}{l}
h_{1}(t) \\
h_{2}(t) \\
h_{3}(t) \\
h_{4}(t)
\end{array}\right)=\left(\begin{array}{c}
\sin (t) \cos (s) \\
\cos ^{2}(t) \\
\cos (t) \\
\cos (t)
\end{array}\right)
\end{gathered}
$$

and

$$
\left(\begin{array}{c}
P_{1}(x, y, u, v) \\
P_{2}(x, y, u, v) \\
P_{3}(x, y, u, v) \\
P_{4}(x, y, u, v)
\end{array}\right)=\left(\begin{array}{c}
x-y+x y \\
x+y-x y \\
x+y+u \\
x+y
\end{array}\right) .
$$

We can easily verify conditions (1.3)

$$
\begin{gathered}
\int_{0}^{2 \pi}\left(\cos ^{2}(s) \sin (s)-\sin (s) \cos ^{2}(s)\right) \mathrm{d} s=0, \\
\int_{0}^{2 \pi}\left(-\sin ^{2}(s) \cos (s)+\cos ^{3}(s)\right) \mathrm{d} s=0, \\
u_{0}=\frac{-3}{10}, \\
v_{0}=-\frac{5}{26} .
\end{gathered}
$$

Computing the functions $\mathcal{F}_{1}, \mathcal{F}_{2}$ we find

$$
\begin{aligned}
& \mathcal{F}_{1}\left(x_{0}, y_{0}\right)=\frac{7}{9}+\frac{7}{6} x_{0}-\frac{4}{3} y_{0}, \\
& \mathcal{F}_{2}\left(x_{0}, y_{0}\right)=\frac{7}{9}+\frac{7}{6} x_{0}+\frac{4}{3} y_{0},
\end{aligned}
$$

The system $\mathcal{F}_{1}=\mathcal{F}_{2}=0$ has one solution $\left(x_{0}^{*}, y_{0}^{*}\right)$ given by $\left(-\frac{2}{3}, 0\right)$ and the eigenvalues of the jacobian matrix of $\left(\begin{array}{l}\mathcal{F}_{1}\left(x_{0}, y_{0}\right) \\ \mathcal{F}_{2}\left(x_{0}, y_{0}\right)\end{array}\right)$ at this solution are 


$$
\begin{aligned}
& \left(\begin{array}{l}
\frac{5}{4}+\frac{1}{12} I \sqrt{233} \\
\frac{5}{4}-\frac{1}{12} I \sqrt{233}
\end{array}\right) \text {, which have two positive real parts. Since } \\
& \operatorname{det}\left(\left.\frac{\partial\left(\mathcal{F}_{1}, \mathcal{F}_{2}\right)}{\partial\left(x_{0}, y_{0}\right)}\right|_{\left(x_{0}, y_{0}\right)=\left(x_{0}^{*}, y_{0}^{*}\right)}\right)=\frac{28}{9} \text {, then the differential system (1.1) has an unsta- } \\
& \text { ble periodic solution }\left(\begin{array}{l}
x(t, \varepsilon) \\
y(t, \varepsilon) \\
u(t, \varepsilon) \\
v(t, \varepsilon)
\end{array}\right) \text {, tending to the unstable periodic solution } \\
& \left(\begin{array}{l}
x(t) \\
y(t) \\
u(t) \\
v(t)
\end{array}\right)=\left(\begin{array}{c}
\frac{-1}{3}-\frac{1}{3} \cos ^{2}(t) \\
\frac{1}{3} \sin (t) \cos (t) \\
\frac{-3}{10} \cos (t)+\frac{1}{10} \sin (t) \\
\frac{-5}{26} \cos (t)+\frac{1}{26} \sin (t)
\end{array}\right)
\end{aligned}
$$

of the differential system

$$
\begin{gathered}
\dot{x}=-y+\sin (t) \cos (t), \\
\dot{y}=x+\cos ^{2}(t), \\
\dot{u}=3 u+\cos (t), \\
\dot{v}=5 v+\cos (t),
\end{gathered}
$$

when $\varepsilon \rightarrow 0$.

\subsection{Application of Theorem 3}

Consider the differential system (1.1) where

$$
\begin{gathered}
\boldsymbol{A}=\left(\begin{array}{cccc}
0 & -1 & 0 & 0 \\
1 & 0 & 0 & 0 \\
0 & 0 & 0 & 0 \\
0 & 0 & 0 & 3
\end{array}\right), \\
\left(\begin{array}{c}
h_{1}(t) \\
h_{2}(t) \\
h_{3}(t) \\
h_{4}(t)
\end{array}\right)=\left(\begin{array}{c}
\sin (t) \cos (s) \\
\sin ^{3}(t) \cos (t) \\
\cos (t) \\
\sin (t)
\end{array}\right)
\end{gathered}
$$

and

$$
\left(\begin{array}{c}
P_{1}(x, y, u, v) \\
P_{2}(x, y, u, v) \\
P_{3}(x, y, u, v) \\
P_{4}(x, y, u, v)
\end{array}\right)=\left(\begin{array}{c}
x-y+x y \\
x+y-x y \\
x+y-u^{2}+3 u \\
x+y
\end{array}\right) .
$$

We can easily verify conditions (1.4) 


$$
\begin{gathered}
\int_{0}^{2 \pi}\left(\cos ^{2}(s) \sin (s)+\sin ^{4}(s) \cos (s)\right) \mathrm{d} s=0 \\
\int_{0}^{2 \pi}\left(-\sin ^{2}(s) \cos (s)+\cos ^{2}(s) \sin ^{3}(s)\right) \mathrm{d} s=0, \\
\int_{0}^{2 \pi} \cos (s) \mathrm{d} s=0, \\
v_{0}=-\frac{1}{10} .
\end{gathered}
$$

Computing the functions $\mathcal{F}_{1}, \mathcal{F}_{2}$ and $\mathcal{F}_{3}$ we find

$$
\begin{gathered}
\mathcal{F}_{1}\left(x_{0}, y_{0}, u_{0}\right)=\frac{15}{16} x_{0}-\frac{49}{48} y_{0}+\frac{127}{720}, \\
\mathcal{F}_{2}\left(x_{0}, y_{0}, u_{0}\right)=\frac{47}{48} x_{0}+\frac{17}{16} y_{0}+\frac{737}{720}, \\
\mathcal{F}_{3}\left(x_{0}, y_{0}, u_{0}\right)=-u_{0}^{3}+3 u_{0}-\frac{1}{2},
\end{gathered}
$$

The system $\mathcal{F}_{1}=\mathcal{F}_{2}=\mathcal{F}_{3}=0$ has two solutions $\left(x_{0}^{*}, y_{0}^{*}, u_{0}^{*}\right)$ given by $\left(-\frac{1}{3},-\frac{2}{15}, \frac{1}{2}(3-\sqrt{7})\right),\left(-\frac{1}{3},-\frac{2}{15}, \frac{1}{2}(3+\sqrt{7})\right)$ and the eigenvalues of the jacobian matrix of $\left(\begin{array}{l}\mathcal{F}_{1}\left(x_{0}, y_{0}, u_{0}\right) \\ \mathcal{F}_{2}\left(x_{0}, y_{0}, u_{0}\right) \\ \mathcal{F}_{3}\left(x_{0}, y_{0}, u_{0}\right)\end{array}\right)$ at these solutions are $\left(\begin{array}{c}1+I \frac{\sqrt{2294}}{48} \\ 1-I \frac{\sqrt{2294}}{48} \\ \sqrt{7}\end{array}\right)$, $\left(\begin{array}{c}1+I \frac{\sqrt{2294}}{48} \\ 1-I \frac{\sqrt{2294}}{48} \\ -\sqrt{7}\end{array}\right)$, which have all at least two positive real parts. Since $\operatorname{det}\left(\left.\frac{\partial\left(\mathcal{F}_{1}, \mathcal{F}_{2}, \mathcal{F}_{3}\right)}{\partial\left(x_{0}, y_{0}, u_{0}\right)}\right|_{\left(x_{0}, y_{0}, u_{0}\right)=\left(x_{0}^{*}, y_{0}^{*}, u_{0}^{*}\right)}\right)$ is $\frac{2299 \sqrt{7}}{1152}, \frac{-2299 \sqrt{7}}{1152}$ respectively, then the differential system (1.1) has two unstable periodic solutions $\left(\begin{array}{l}x_{k}(t, \varepsilon) \\ y_{k}(t, \varepsilon) \\ u_{k}(t, \varepsilon) \\ v_{k}(t, \varepsilon)\end{array}\right)$ with $k=1,2$, tending to the unstable periodic solutions

$$
\left(\begin{array}{l}
x_{1}(t) \\
y_{1}(t) \\
u_{1}(t) \\
v_{1}(t)
\end{array}\right)=\left(\begin{array}{c}
\frac{1}{3}+\frac{1}{5} \sin (t) \cos (t)-\frac{2}{3} \cos ^{2}(t)-\frac{1}{15} \sin (t) \cos ^{3}(t) \\
\frac{1}{5}-\frac{1}{3} \sin (t) \cos (t)-\frac{3}{5} \cos ^{2}(t)+\frac{4}{15} \cos ^{4}(t) \\
\frac{1}{2}(3-\sqrt{7})+\sin (t) \\
\frac{1}{10} \cos (t)+\frac{3}{10} \sin (t)
\end{array}\right)
$$




$$
\left(\begin{array}{l}
x_{2}(t) \\
y_{2}(t) \\
u_{2}(t) \\
v_{2}(t)
\end{array}\right)=\left(\begin{array}{c}
\frac{1}{3}+\frac{1}{5} \sin (t) \cos (t)-\frac{2}{3} \cos ^{2}(t)-\frac{1}{15} \sin (t) \cos ^{3}(t) \\
\frac{1}{5}-\frac{1}{3} \sin (t) \cos (t)-\frac{3}{5} \cos ^{2}(t)+\frac{4}{15} \cos ^{4}(t) \\
\frac{1}{2}(3+\sqrt{7})-\sin (t) \\
\frac{1}{10} \cos (t)+\frac{3}{10} \sin (t)
\end{array}\right)
$$

of the differential system

$$
\begin{gathered}
\dot{x}=-y+\sin (t) \cos (t), \\
\dot{y}=x+\sin ^{3}(t) \cos (t), \\
\dot{u}=\cos (t), \\
\dot{v}=3 v+\sin (t)
\end{gathered}
$$

when $\varepsilon \rightarrow 0$.

\subsection{Application of Theorem 4}

Consider the differential system (1.1) where

$$
\begin{gathered}
\boldsymbol{A}=\left(\begin{array}{cccc}
0 & -1 & 0 & 0 \\
1 & 0 & 0 & 0 \\
0 & 0 & 0 & 0 \\
0 & 0 & 0 & 0
\end{array}\right), \\
\left(\begin{array}{l}
h_{1}(t) \\
h_{2}(t) \\
h_{3}(t) \\
h_{4}(t)
\end{array}\right)=\left(\begin{array}{c}
\sin (t) \\
\cos (t) \\
\sin (t) \\
\sin (t) \cos (t)
\end{array}\right)
\end{gathered}
$$

and

$$
\left(\begin{array}{c}
P_{1}(x, y, u, v) \\
P_{2}(x, y, u, v) \\
P_{3}(x, y, u, v) \\
P_{4}(x, y, u, v)
\end{array}\right)=\left(\begin{array}{c}
3 x^{2}-x y^{2}+y^{2} \\
3 y^{2}-y x^{2}+x^{2} \\
u \\
v
\end{array}\right) .
$$

We can easily verify conditions (1.5)

$$
\begin{gathered}
\int_{0}^{2 \pi}(2 \sin (s) \cos (s)) \mathrm{d} s=0, \\
\int_{0}^{2 \pi}\left(-\sin ^{2}(s)+\cos ^{2}(s)\right) \mathrm{d} s=0, \\
\int_{0}^{2 \pi} \sin (s) \mathrm{d} s=0, \\
\int_{0}^{2 \pi} \sin (s) \cos (s) \mathrm{d} s=0 .
\end{gathered}
$$

Computing the functions $\mathcal{F}_{1} \quad \mathcal{F}_{2} \quad \mathcal{F}_{3}$ and $\mathcal{F}_{4}$ we find

$$
\begin{gathered}
\mathcal{F}_{1}\left(x_{0}, y_{0}, u_{0}, v_{0}\right)=-\frac{1}{8} x_{0}-\frac{3}{8} x_{0}^{2}-\frac{1}{8} y_{0}^{2}-\frac{1}{4} x_{0}^{3}-\frac{1}{4} x_{0} y_{0}^{2}, \\
\mathcal{F}_{2}\left(x_{0}, y_{0}, u_{0}, v_{0}\right)=-\frac{1}{4} x_{0} y_{0}-\frac{1}{4} x_{0}^{2} y_{0}-\frac{1}{4} y_{0}^{3}-\frac{3}{8} y_{0},
\end{gathered}
$$




$$
\begin{aligned}
& \mathcal{F}_{3}\left(x_{0}, y_{0}, u_{0}, v_{0}\right)=u_{0}+1, \\
& \mathcal{F}_{4}\left(x_{0}, y_{0}, u_{0}, v_{0}\right)=v_{0}+\frac{1}{4}
\end{aligned}
$$

The system $\mathcal{F}_{1}=\mathcal{F}_{2}=\mathcal{F}_{3}=\mathcal{F}_{4}=0$ has three solutions $\left(x_{0}^{*}, y_{0}^{*}, u_{0}^{*}, v_{0}^{*}\right)$ given by $\left(0,0,-1, \frac{-1}{4}\right),\left(-\frac{1}{2}, 0,-1,-\frac{1}{4}\right),\left(-1,0,-1,-\frac{1}{4}\right)$ and the eigenvalues of the jacobian matrix of $\left(\begin{array}{l}\mathcal{F}_{1}\left(x_{0}, y_{0}, u_{0}, v_{0}\right) \\ \mathcal{F}_{2}\left(x_{0}, y_{0}, u_{0}, v_{0}\right) \\ \mathcal{F}_{3}\left(x_{0}, y_{0}, u_{0}, v_{0}\right) \\ \mathcal{F}_{4}\left(x_{0}, y_{0}, u_{0}, v_{0}\right)\end{array}\right)$ at these solutions are $\left(\begin{array}{c}\frac{-1}{8} \\ \frac{-3}{8} \\ 1 \\ 1\end{array}\right),\left(\begin{array}{c}\frac{1}{16} \\ \frac{-5}{16} \\ 1 \\ 1\end{array}\right)$, $\left(\begin{array}{c}\frac{-1}{8} \\ \frac{-3}{8} \\ 1 \\ 1\end{array}\right)$, which have all at least two positive real parts. Since
\[ \operatorname{det}\left(\left.\frac{\partial\left(\mathcal{F}_{1}, \mathcal{F}_{2}, \mathcal{F}_{3}, \mathcal{F}_{4}\right)}{\partial\left(x_{0}, y_{0}, u_{0}, v_{0}\right)}\right|_{\left(x_{0}, y_{0}, u_{0}, v_{0}\right)=\left(x_{0}^{*}, y_{0}^{*}, u_{0}^{*}, v_{0}^{*}\right)}\right) \]

at these three solutions $\left(x_{0}^{*}, y_{0}^{*}, u_{0}^{*}, v_{0}^{*}\right)$ is $\frac{3}{64},-\frac{5}{256}, \frac{3}{64}$, respectively, then this differential system has three unstable periodic solutions $\left(\begin{array}{l}x_{k}(t, \varepsilon) \\ y_{k}(t, \varepsilon) \\ u_{k}(t, \varepsilon) \\ v_{k}(t, \varepsilon)\end{array}\right)$, where $k=1$,

2, 3 tending to the unstable periodic solutions

$$
\begin{aligned}
& \left(\begin{array}{l}
x_{1}(t) \\
y_{1}(t) \\
u_{1}(t) \\
v_{1}(t)
\end{array}\right)=\left(\begin{array}{c}
-\cos (t) \\
0 \\
-\cos (t) \\
\frac{1}{4}-\frac{1}{2} \cos ^{2}(t)
\end{array}\right) \\
& \left(\begin{array}{l}
x_{2}(t) \\
y_{2}(t) \\
u_{2}(t) \\
v_{2}(t)
\end{array}\right)=\left(\begin{array}{c}
0 \\
\sin (t) \\
-\cos (t) \\
\frac{1}{4}-\frac{1}{2} \cos { }^{2}(t)
\end{array}\right) \\
& \left(\begin{array}{l}
x_{3}(t) \\
y_{3}(t) \\
u_{3}(t) \\
v_{3}(t)
\end{array}\right)=\left(\begin{array}{c}
-\frac{1}{2} \cos (t) \\
\frac{1}{2} \sin (t) \\
-\cos (t) \\
\frac{1}{4}-\frac{1}{2} \cos (t)
\end{array}\right)
\end{aligned}
$$


of the differential system

$$
\begin{gathered}
\dot{x}=-y+\sin (t), \\
\dot{y}=x+\cos (t), \\
\dot{u}=\sin (t), \\
\dot{v}=\sin (t) \cos (t),
\end{gathered}
$$

when $\varepsilon \rightarrow 0$.

\subsection{Application of Theorem 5}

Consider the differential system (1.1) where

$$
\begin{gathered}
A=\left(\begin{array}{cccc}
0 & -1 & 0 & 0 \\
1 & 0 & 0 & 0 \\
0 & 0 & 2 & 1 \\
0 & 0 & 0 & 2
\end{array}\right), \\
\left(\begin{array}{l}
h_{1}(t) \\
h_{2}(t) \\
h_{3}(t) \\
h_{4}(t)
\end{array}\right)=\left(\begin{array}{c}
-\sin (t) \\
-\cos (t) \\
\sin (t) \\
\sin (t)
\end{array}\right)
\end{gathered}
$$

and

$$
\left(\begin{array}{c}
P_{1}(x, y, u, v) \\
P_{2}(x, y, u, v) \\
P_{3}(x, y, u, v) \\
P_{4}(x, y, u, v)
\end{array}\right)=\left(\begin{array}{c}
y-x^{2} y \\
-x y^{2} \\
x+y+u \\
x+y
\end{array}\right) .
$$

We can easily verify conditions (1.6)

$$
\begin{gathered}
\int_{0}^{2 \pi}(-2 \cos (s) \sin (s)) \mathrm{d} s=0 \\
\int_{0}^{2 \pi}\left(\sin ^{2}(s)-\cos ^{2}(s)\right) \mathrm{d} s=0 \\
u_{0}=-\frac{1}{25} \\
v_{0}=-\frac{1}{5}
\end{gathered}
$$

Computing the functions $\mathcal{F}_{1}$ and $\mathcal{F}_{2}$ we find

$$
\begin{gathered}
\mathcal{F}_{1}\left(x_{0}, y_{0}\right)=-\frac{7}{8} y_{0}-\frac{6}{8} x_{0} y_{0}, \\
\mathcal{F}_{2}\left(x_{0}, y_{0}\right)=\frac{1}{2}-\frac{5}{8} x_{0}+\frac{1}{8} x_{0}^{2}-\frac{5}{8} y_{0}^{2},
\end{gathered}
$$

The system $\mathcal{F}_{1}=\mathcal{F}_{2}=0$ has two solutions $\left(x_{0}^{*}, y_{0}^{*}\right)$ given by $(1,0),(4,0)$ and the eigenvalues of the jacobian of $\left(\begin{array}{l}\mathcal{F}_{1}\left(x_{0}, y_{0}\right) \\ \mathcal{F}_{2}\left(x_{0}, y_{0}\right)\end{array}\right)$ at these solutions are 
$\left(\begin{array}{l}\frac{1}{8} I \sqrt{3} \\ \frac{-1}{8} I \sqrt{3}\end{array}\right),\left(\begin{array}{c}\frac{1}{8} I \sqrt{51} \\ \frac{-1}{8} I \sqrt{51}\end{array}\right)$, which have all zero real parts. Since
$\operatorname{det}\left(\left.\frac{\partial\left(\mathcal{F}_{1}, \mathcal{F}_{2}\right)}{\partial\left(x_{0}, y_{0}\right)}\right|_{\left(x_{0}, y_{0}\right)=\left(x_{0}^{*}, y_{0}^{*}\right)}\right)$ for these solutions $\left(x_{0}^{*}, y_{0}^{*}\right)$ is $\frac{3}{64}, \frac{51}{64}$, respectively, then this differential system has two periodic solutions $\left(\begin{array}{c}x(t, \varepsilon) \\ y(t, \varepsilon) \\ u(t, \varepsilon) \\ v(t, \varepsilon)\end{array}\right)$, tending to the two periodic solutions

$$
\begin{aligned}
& \left(\begin{array}{l}
x_{1}(t) \\
y_{1}(t) \\
u_{1}(t) \\
v_{1}(t)
\end{array}\right)=\left(\begin{array}{c}
\cos (t) \\
0 \\
-\frac{1}{25} \cos (t)-\frac{7}{25} \sin (t) \\
-\frac{1}{5} \cos (t)-\frac{2}{5} \sin (t)
\end{array}\right) \\
& \left(\begin{array}{l}
x_{2}(t) \\
y_{2}(t) \\
u_{2}(t) \\
v_{2}(t)
\end{array}\right)=\left(\begin{array}{c}
4 \cos (t) \\
3 \sin (t) \\
\frac{-1}{25} \cos (t)-\frac{7}{25} \sin (t) \\
-\frac{1}{5} \cos (t)-\frac{2}{5} \sin (t)
\end{array}\right)
\end{aligned}
$$

of the differential system

$$
\begin{gathered}
\dot{x}=-y-\sin (t), \\
\dot{y}=x-\cos (t), \\
\dot{u}=2 u+v+\sin (t), \\
\dot{v}=2 v+\sin (t)
\end{gathered}
$$

when $\varepsilon \rightarrow 0$.

In this case we can say nothing about the stability of these solutions.

\section{Conclusion}

This study leads us to consider the general case when $\mathrm{A}$ is an $n \times n$ matrix, $P_{1} \cdots P_{n}$ are polynomials in the variables $x_{1}, \cdots, x_{n}$ of degree $n$ and $h_{i}(t+2 \pi)=h_{i}(t)$, with $i=1 \cdots n$. In the next work, we shall generalize the studied system (1.1) in $\mathbb{R}^{n}$.

\section{References}

[1] Champneys, A.R. (1998) Homoclinic Orbits in Reversible Systems and Their Applications in Mechanics, Fluids and Optics. Physica D, 112, 158-186. https://doi.org/10.1016/S0167-2789(97)00209-1 
[2] Chaparova, J. (2002) Existence and Numerical Approximations of Periodic Solutions of Semilinear Fourth-Order Differential Equations. Journal of Mathematical Analysis and Applications, 273, 121-136. https://doi.org/10.1016/S0022-247X(02)00216-0

[3] Conti, M., Terracini, S. and Verzini, G. (2003) Infinitely Many Solutions to Fourth Order Superlinear Periodic Problems. Transactions of the American Mathematical Society, 356, 3283-3300. https://doi.org/10.1090/S0002-9947-03-03514-1

[4] Ezeilo, J.O.C. and Tejumola, H.O. (1979) Periodic Solutions of a Certain Fourth Order Differential Equation. Atti della Accademia Nazionale dei Lincei. Classe di Scienze Fisiche, Matematiche e Naturali, 66, 344-350.

[5] Feng, C.H. (1995) On the Existence of Periodic Solutions to Certain Fourth Differential Equation. Annals of Differential Equations, 11, 46-50.

[6] Tejumola, H.O. (1968) Boundedess and Periodicity of Solutions of Certain FourthOrder Differential Equations. Annali di Matematica Pura ed Applicata, 80, 177-196. https://doi.org/10.1007/BF02413628

[7] Tejumola, H.O. (1974) Periodic Solutions of Certain Fourth-Order Differential Equations. Atti della Accademia Nazionale dei Lincei. Classe di Scienze Fisiche, Matematiche e Naturali, 57, 328-336.

[8] Tejumola, H.O. (1974) On the Existence of Periodic Solutions of Certain FourthOrder Differential Equations. Atti della Accademia Nazionale dei Lincei. Classe di Scienze Fisiche, Matematiche e Naturali, 57, 530-533.

[9] Tejumola, H.O. (1975) On a Certain Nonlinear Fourth Order Ordinary Differential Equation. Atti della Accademia Nazionale dei Lincei. Classe di Scienze Fisiche, Matematiche e Naturali, 58, 137-142.

[10] Tiryaki, A. (1989) Periodic Solutions of a Certain Fourth Order Differential Equations. Indian Journal of Pure and Applied Mathematics, 20, 235-241.

[11] Tunç, E. (2005) On the Periodic Solutions of Certain Fourth and Fifth Order Vector Differential Equations. Mathematical Communications, 10, 135-141.

[12] Llibre, J., Novas, D.D. and Teixeira, M.A. (2014) Higher Order Averaging Theory for Finding Periodic Solutions via Brouwer Degree. Nonlinearity, 27, 563-583. https://doi.org/10.1088/0951-7715/27/3/563

[13] Fatou, P. (1928) Sur le mouvement d'un système soumis à des forces à courte période. Bulletin de la Société Mathématique de France, 56, 98-139.

[14] Bogoliubov, N.N. and Krylov, N. (1934) The Application of Methods of Nonlinear Mechanics in the Theory of Stationary Oscillations. Publ. 8 of the Ukrainian Academy of Sciences, Kiev.

[15] Bogoliubov, N.N. (1945) On Some Statistical Methods in Mathematical Physics. Izv.vo Akademiiānauk Ukrainskoĩ SSR, Kiev.

[16] Bogoliubov, N.N. and Mitropolsky, Yu.A. (1961) Asymtotic Methods in the Theory of Nonlinear Oscillations. Gordon and Breach, New York.

[17] Sanders, J.A. and Verhulst, F. (1985) Averaging Methods in Nonlinear Dynamical Systems. Applied Mathematical Sciences, 59, Springer, Berlin. https://doi.org/10.1007/978-1-4757-4575-7

[18] Llibre, J. and Makhlouf, A. (2009) Bifurcation of Limit Cycles from a 4-Dimensional Center in 1:n Resonance. Applied Mathematics and Computation, 215, 140-146. https://doi.org/10.1016/j.amc.2009.04.065

[19] Llibre, J., Sellami, N. and Makhlouf, A. (2009) Limit Cycles for a Class of FourthOrder Differential Equations. Applicable Analysis, 88, 1617-1630.

https://doi.org/10.1080/00036810903114833 
[20] Llibre, J. and Makhlouf, A. (2012) Limit Cycles for Fourth-Order Autonomous Differential Equations. Journal EJDE, 2012, 1-17.

[21] Llibre, J. and Makhlouf, A. (2012) On the Limit Cycles for a Class of Fourth-Order Differential Equations. Journal of Physics A: Mathematical and Theoretical, 45, Article ID: 055214. https://doi.org/10.1088/1751-8113/45/5/055214

[22] Llibre, J. and Makhlouf, A. (2012) Periodic Solutions of the Fourth-Order NonAutonomous Differential Equation $u^{\prime \prime \prime \prime}+q u^{\prime \prime}+p u=\varepsilon f\left(t, u, u^{\prime}, u^{\prime \prime}, u^{\prime \prime \prime}, u^{\prime \prime \prime}\right)$. Applied Mathematics and Computation, 219, 827-836. https://doi.org/10.1016/j.amc.2012.06.047

[23] Makhlouf, A. and Bousbiat, L. (2015) Periodic Solutions of Some Polynomial Differential System in Dimension 3 via Averaging Theory. International Journal of Differential Equations, 2015, Article ID: 263837. (In Hindawi) https://doi.org/10.1155/2015/263837

[24] Vehrulst, F. (1996) Nonlinear Differential Equations and Dynamical Systems. Universitext, Springer, Berlin. https://doi.org/10.1007/978-3-642-61453-8

[25] Malkin, I.G. (1956) Some Problems of the Theory of Nonlinear Oscillations. Gosudarstv. Izdat. Tehn.-Teor. Lit., Moscow. (In Russian)

[26] Roseau, M. (1966) Vibrations Non Linéaires et théorie de la stabilité. Springer Tracts in Natural Philosophy, Vol. 8, Springer-Verlag, Berlin-New York. (In French)

[27] Buica, A., Francoise, J.P. and Llibre, J. (2007) Periodic Solutions of Nonlinear Periodic Differential Systems with a Small Parameter. Communications on Pure and Applied Analysis, 6, 103-111.

\section{Submit or recommend next manuscript to SCIRP and we will provide best} service for you:

Accepting pre-submission inquiries through Email, Facebook, LinkedIn, Twitter, etc. A wide selection of journals (inclusive of 9 subjects, more than 200 journals)

Providing 24-hour high-quality service

User-friendly online submission system

Fair and swift peer-review system

Efficient typesetting and proofreading procedure

Display of the result of downloads and visits, as well as the number of cited articles

Maximum dissemination of your research work

Submit your manuscript at: http://papersubmission.scirp.org/

Or contact jamp@scirp.org 